\title{
The Effect of Sleep on Response to Happy and Sad Images
}

\section{Ateke Goshvarpour, Ataollah Abbasi*, Atefeh Goshvarpour}

Department of Biomedical Engineering, Faculty of Electrical Engineering, Sahand University of Technology, Tabriz, Iran

Article Info:

Received: 28 Apr 2016

\section{A BSTRACT}

Introduction: Studies show that changes in the average of sleep duration has unpleasant effects on physiological and cognitive functions of individuals. On the other hand, recording and analysis of biological signals are one of the safest methods for studying emotional responses, that participant is rarely able to fake them voluntarily. Given that the effects of sleep on the physiological parameters of individuals in response to emotion is not considered sufficiently, this study has attempted to provide a comprehensive evaluation on signal analysis methods, to examine the differences in autonomic parameters, including the electrocardiogram and galvanic skin response (GSR). Materials and Methods: Autonomic signals of 47 students while watching the happy and sad images were collected and analyzed. For this purpose, a wide range of linear and nonlinear characteristics were extracted from the signals and the differences between the two groups were evaluated by means of the non-parametric Mann-Whitney test. Results: 22 of the participants, had normal sleep (mean age 21.32 \pm 1.67 years) while 25 subjects had insufficient sleep (mean age $21.88 \pm 1.64$ years). The results of present study show that physiological responses to emotional stimuli depend on the duration of sleep. The highest percentage of discrimination devote to the non-linear characteristics of GSR signal in response to the sad images. of these, the Poincare indices revealed significant differences between the two groups. The results of linear analysis also indicated better performance of GSR signal in illustrating the difference between emotional responses of two groups with different amount of sleep. Conclusion: There is a significant relation between the amount of sleep and emotional behavior of the participants. Analysis of autonomic parameters enable us to investigate this relationship. However, the results are depended on the type of stimulation and the methodology applied for data analysis.

\section{Key words:}

1. Emotions

2. Nonlinear Dynamics

3. Sleep

*Corresponding Author: Ataollah Abbasi

E-mail: ata.abbasi@sut.ac.ir 


\section{تأثير خواب در پاسخ به تصاوير شاد و غم}

\section{عاتكه گشواريور، عطاله عباسى"، عاطفه كشواريور}

كروه مهندسى يزشكى، دانشكده مهندسى برق، دانشكاه صنعتى سهند، تبريز، ايران

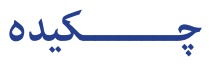

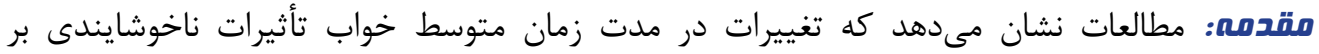

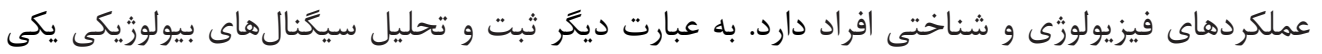

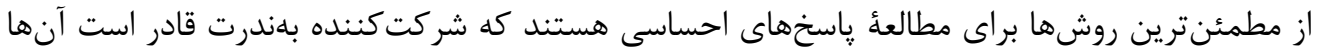

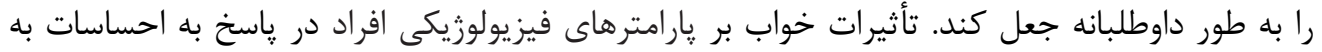

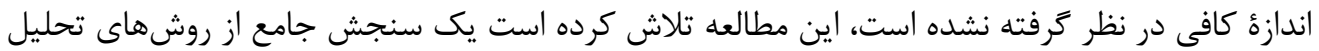

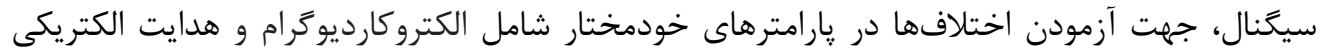

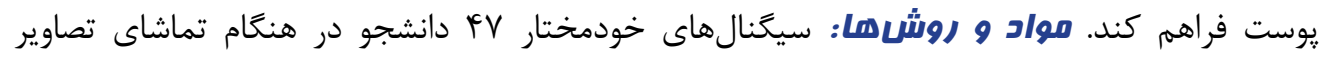

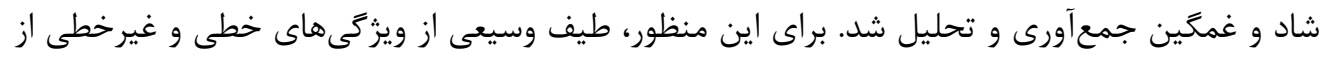

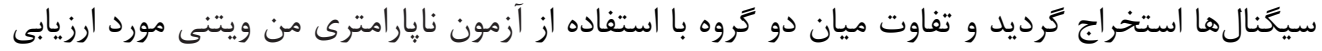

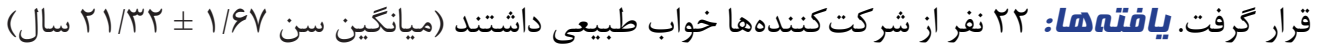

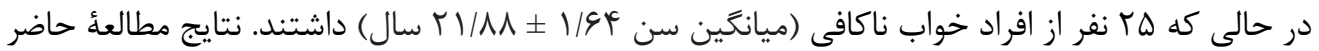

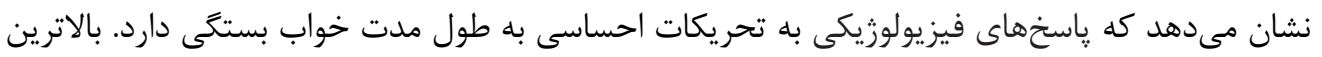

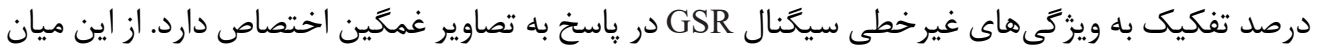

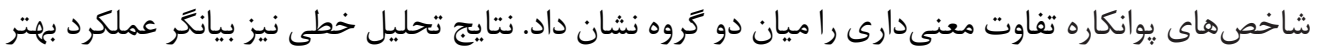

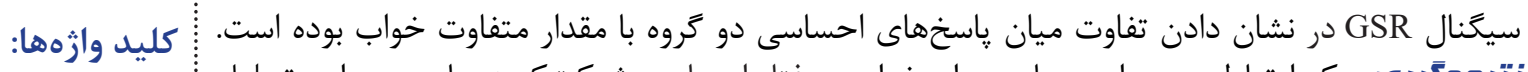

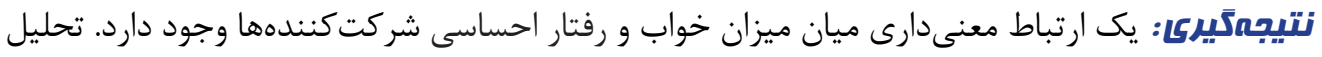

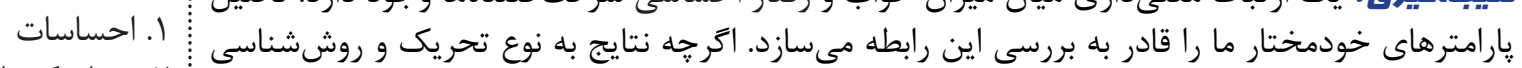

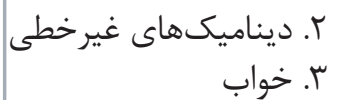

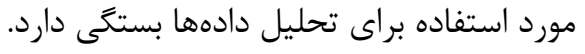




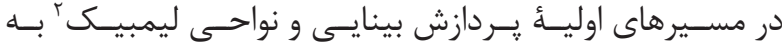

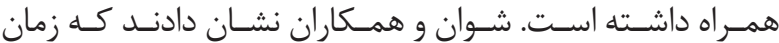

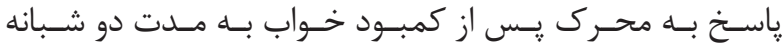

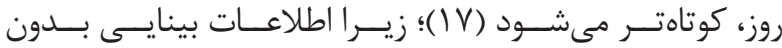

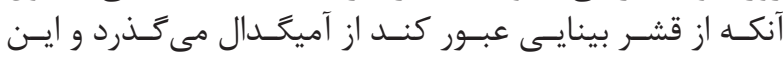

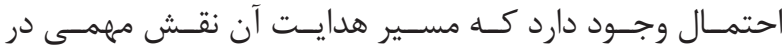

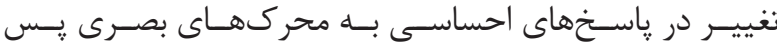
از اختـلال خـواب داشـته باشـــ (19، 19 (19).

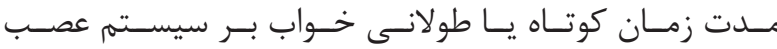

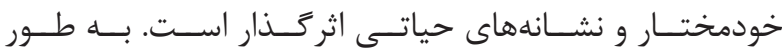

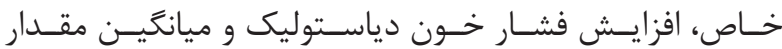

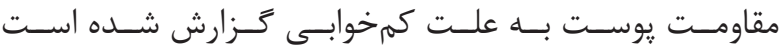

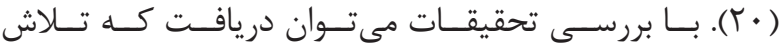

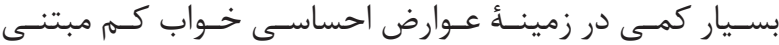

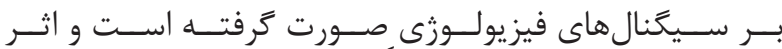

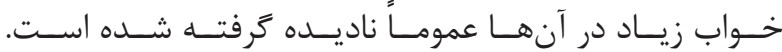

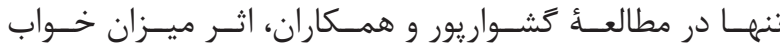

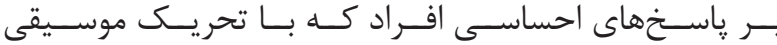

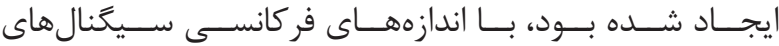

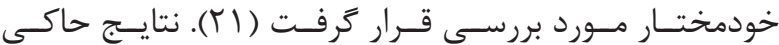

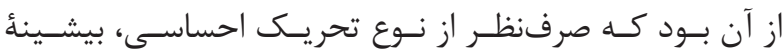

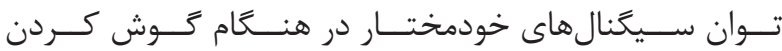

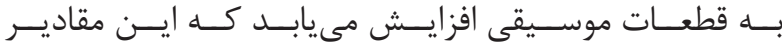

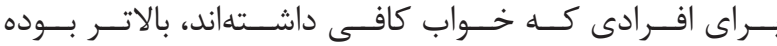

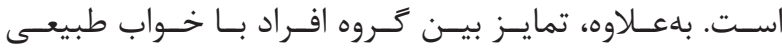

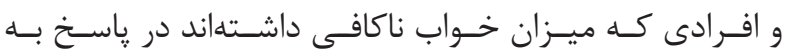

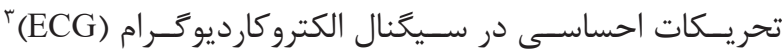

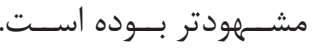

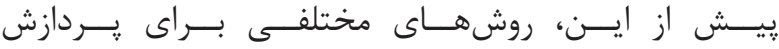

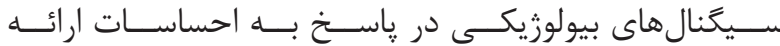

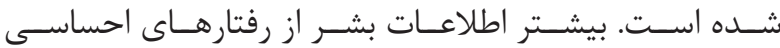

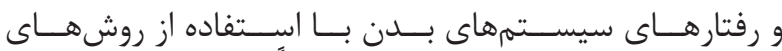

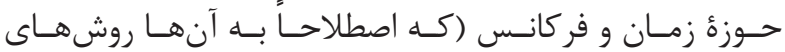

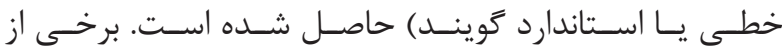

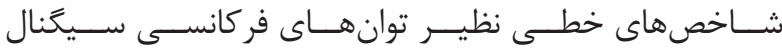

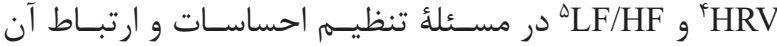

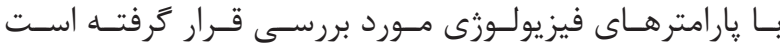

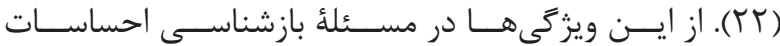

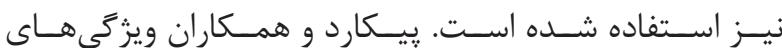

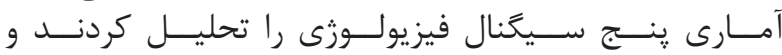

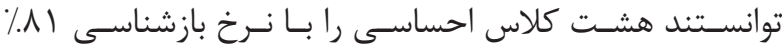

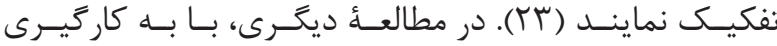

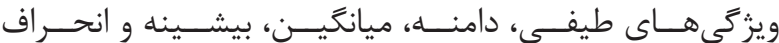

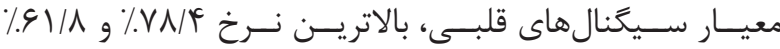

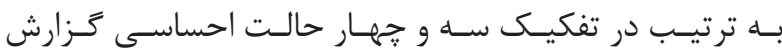

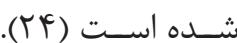

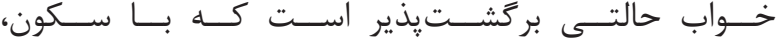

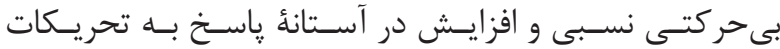

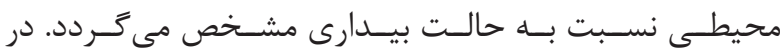

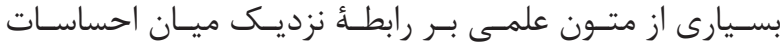

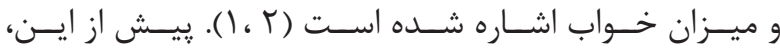

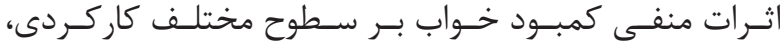

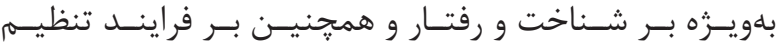

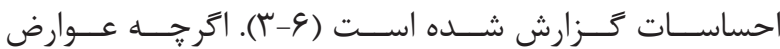

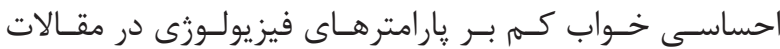

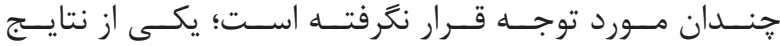

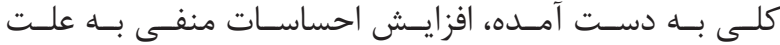

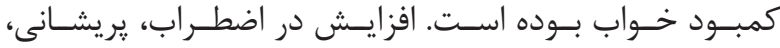

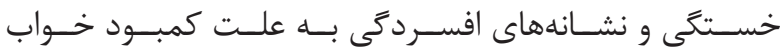

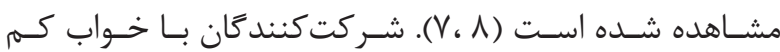

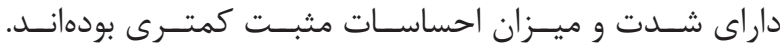

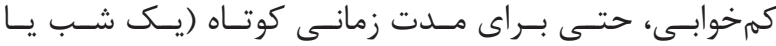

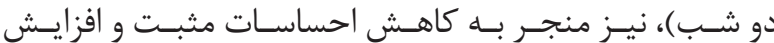

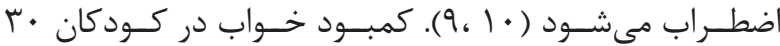

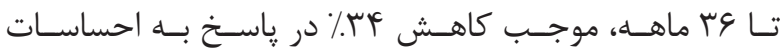

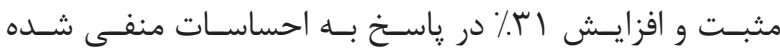

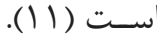

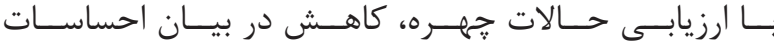

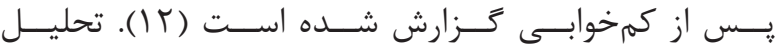

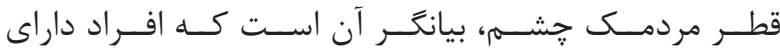

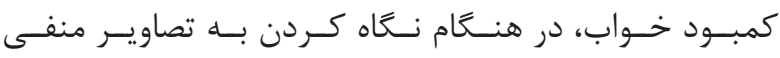

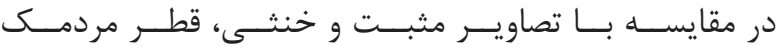

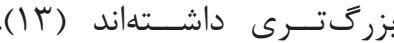

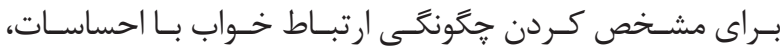

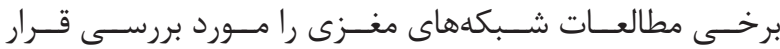

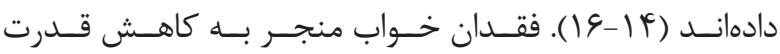

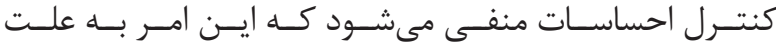

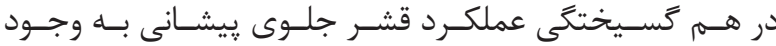

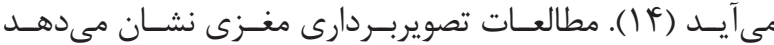

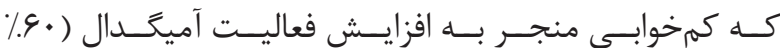

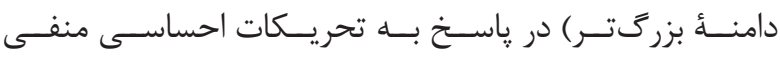

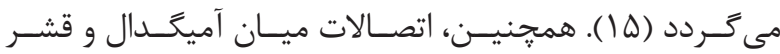

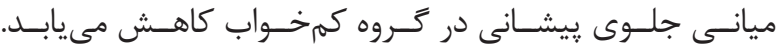

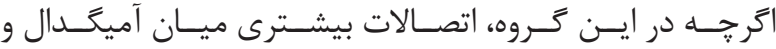

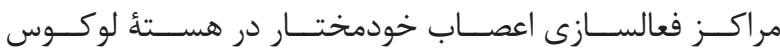

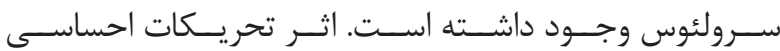

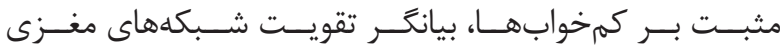

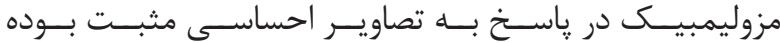

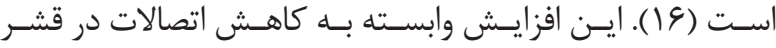

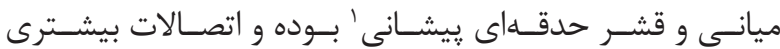

${ }^{5}$ Low-frequency to high-frequency ratio 


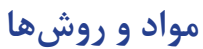

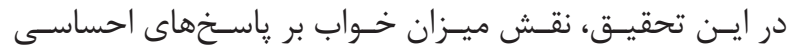

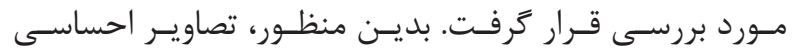

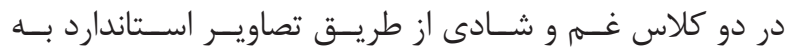

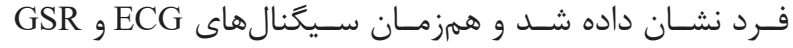

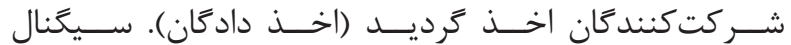

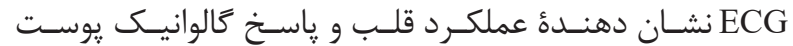

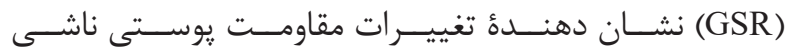

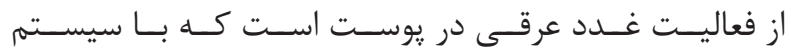

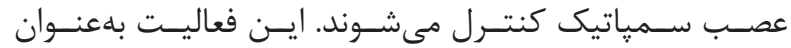

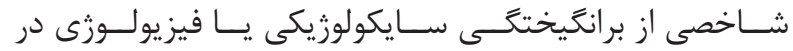

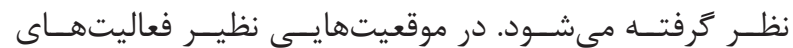

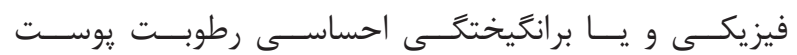

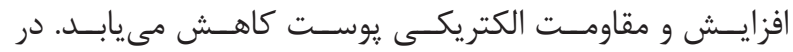

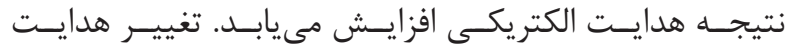

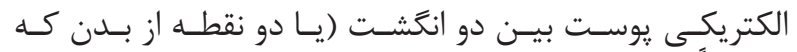

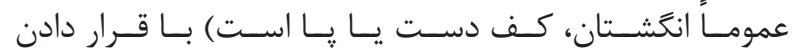

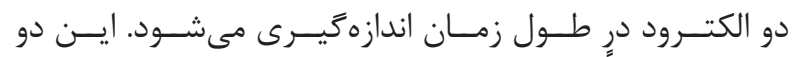

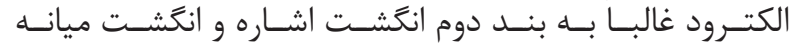

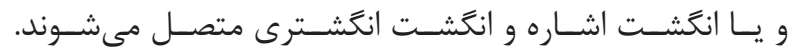

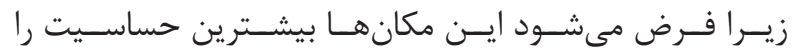

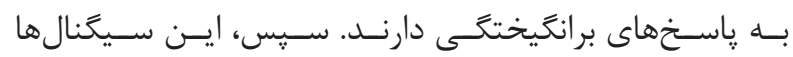

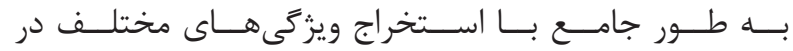

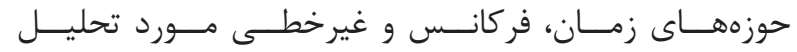

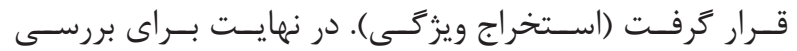

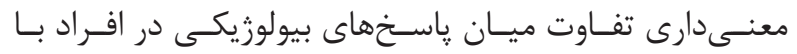

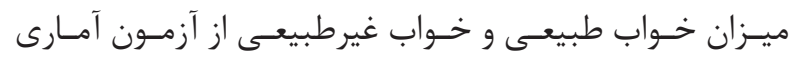

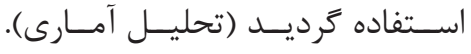

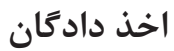

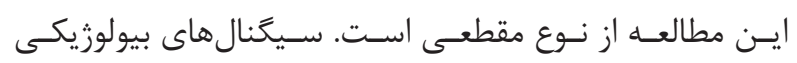

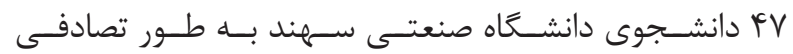

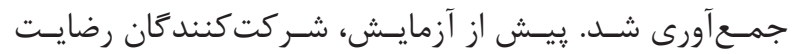

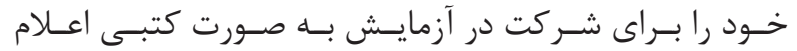

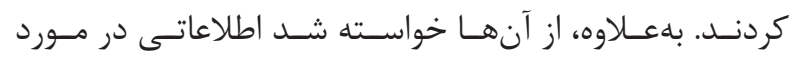

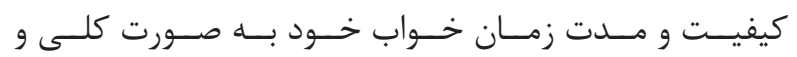

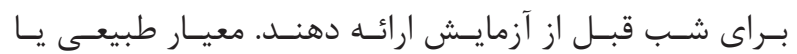

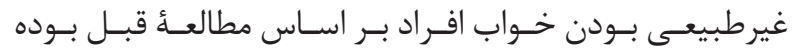

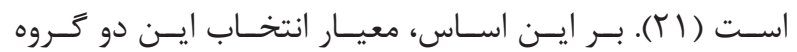

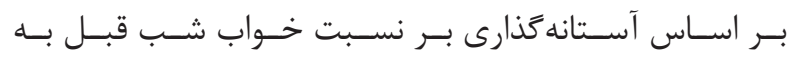

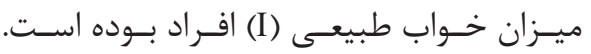

\section{$I=\frac{\text { previous night sleep hours of the subject }}{\text { general night sleep hours of the subject }}$}

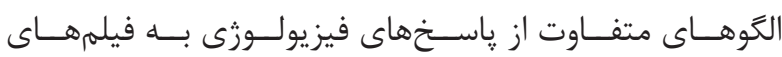

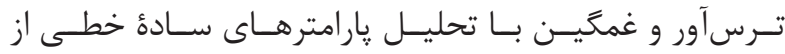

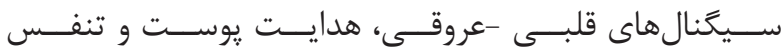

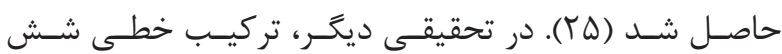

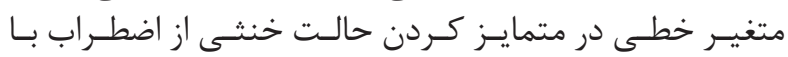

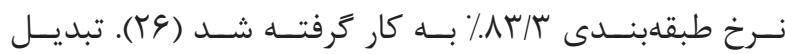

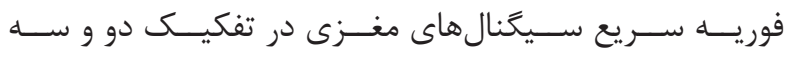

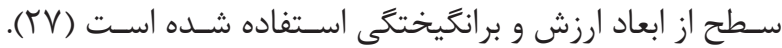

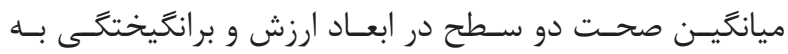

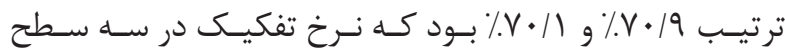

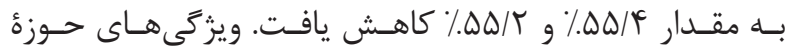

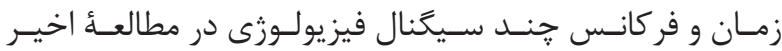

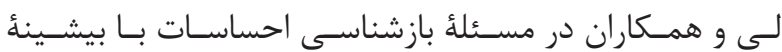

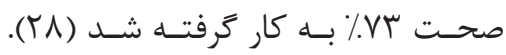

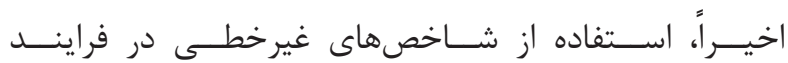

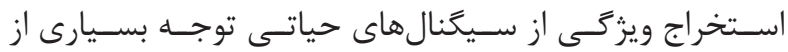

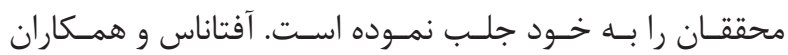

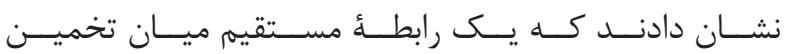
ديناميـك קِيجيدگ

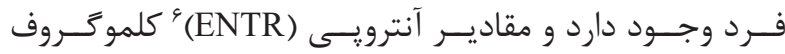

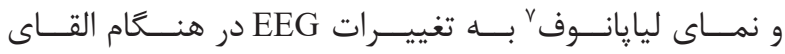

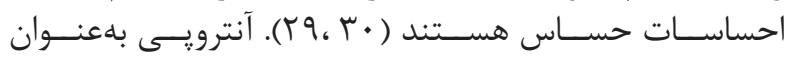

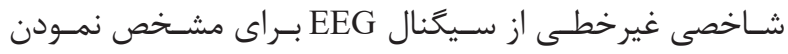

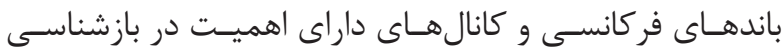

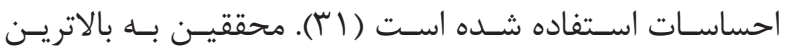

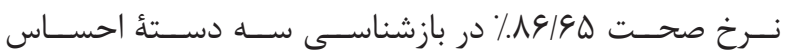

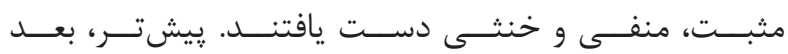

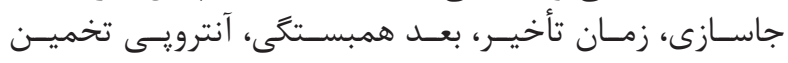

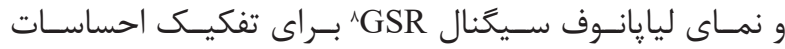

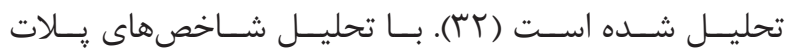

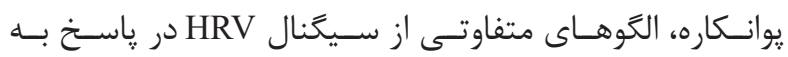

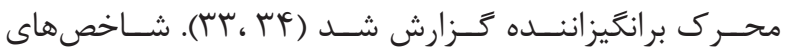

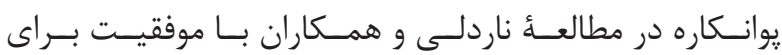

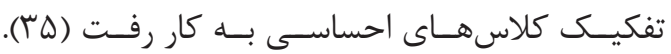

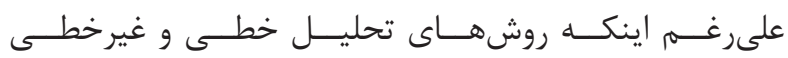

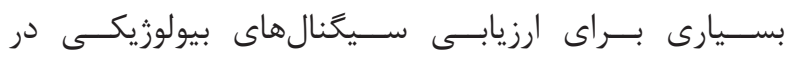

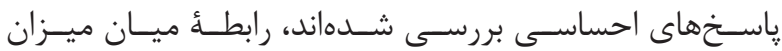

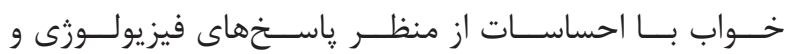

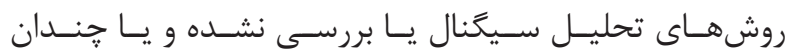

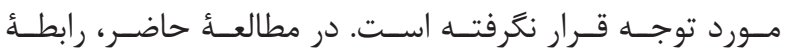

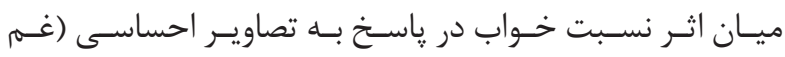

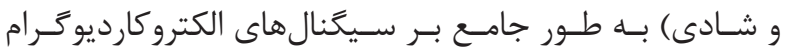

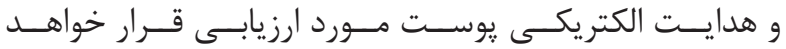

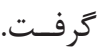




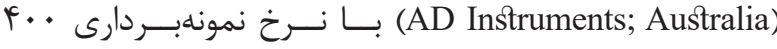

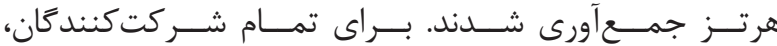

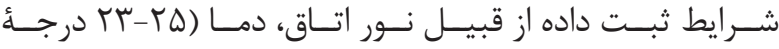

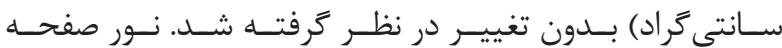

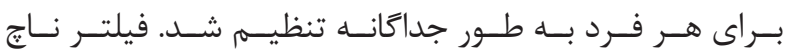

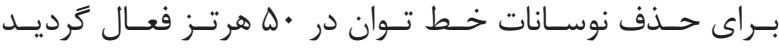

(rV)

\section{استخراج ويثزى

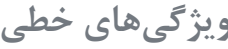

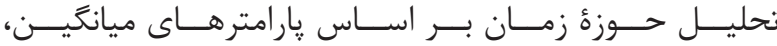

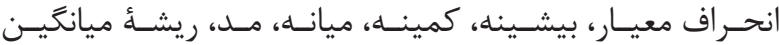

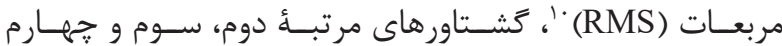

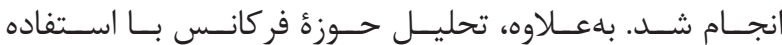

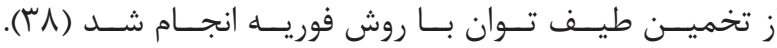

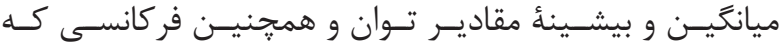

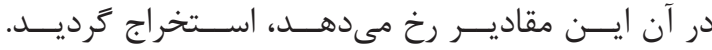

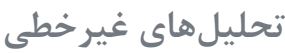

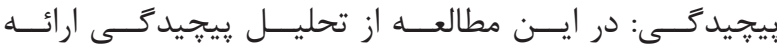

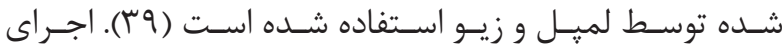

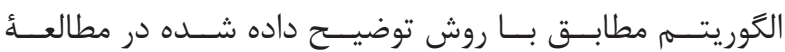

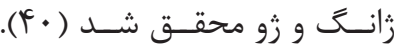

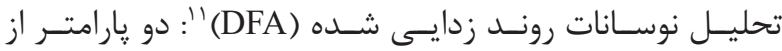

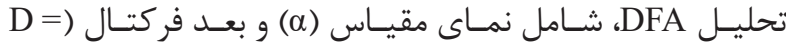

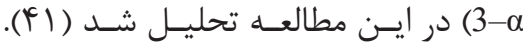

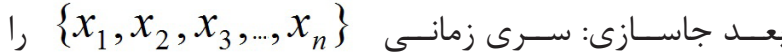

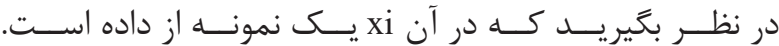

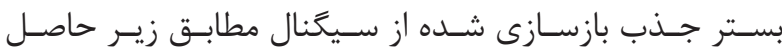

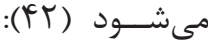

$$
\underline{X}(i)=\left\{x_{i}, x_{i+\tau}, x_{i+2 \tau}, \ldots, x_{i+(m-1) \tau}\right\}
$$

كه در آن آتأخير محاط و m بعد جاسازى است.

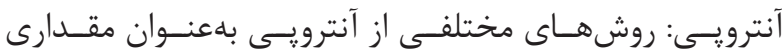

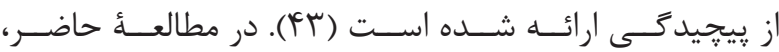

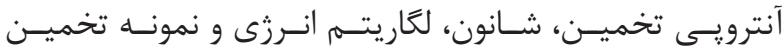

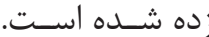

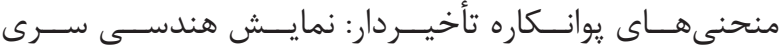

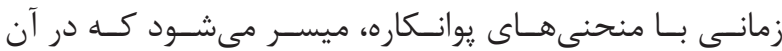

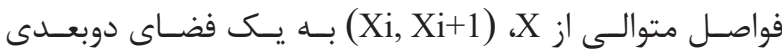

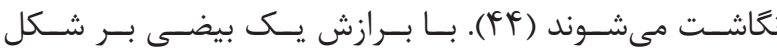

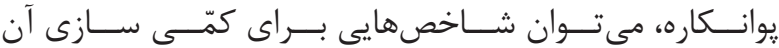

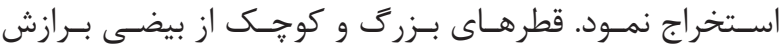

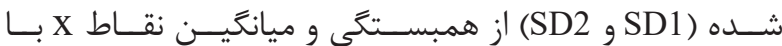

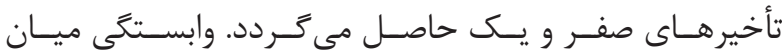

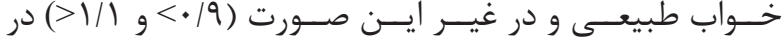

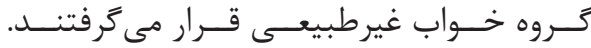

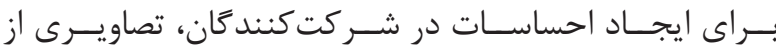

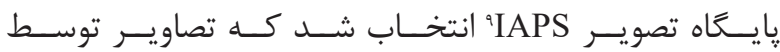

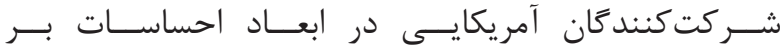

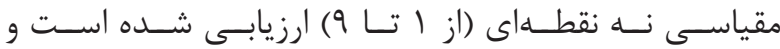

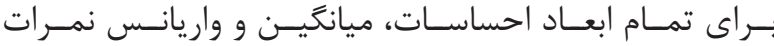

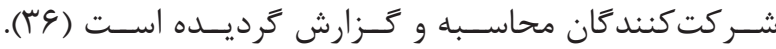

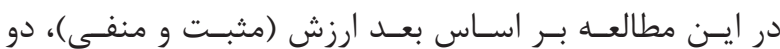

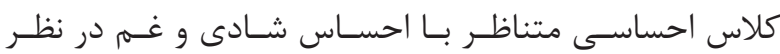

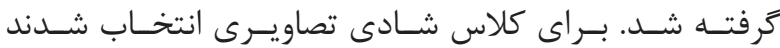

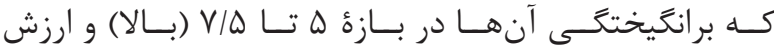

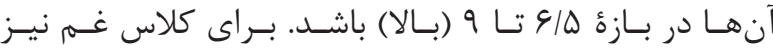

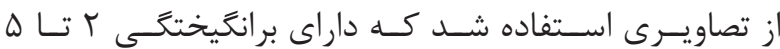

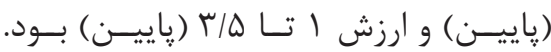

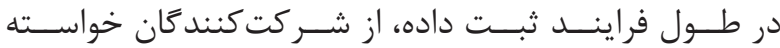

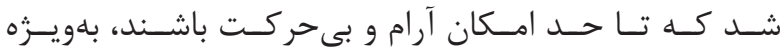

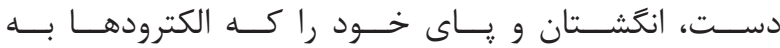

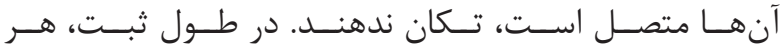

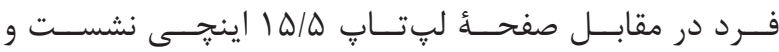

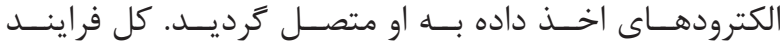

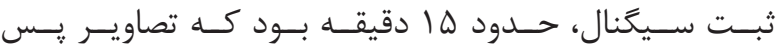

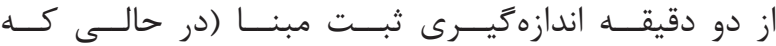

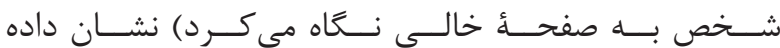

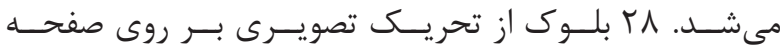

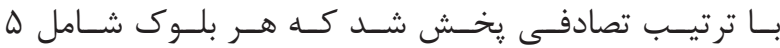

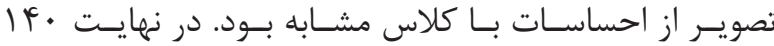

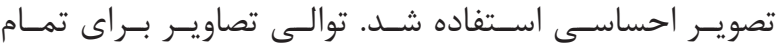

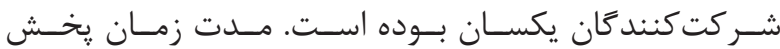

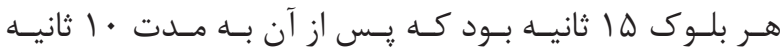

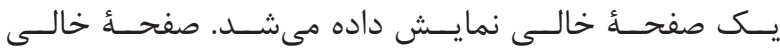

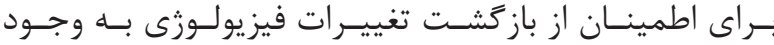

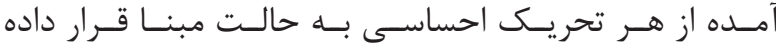

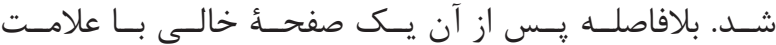

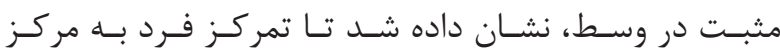

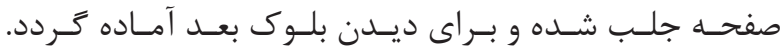

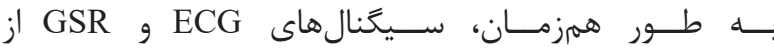

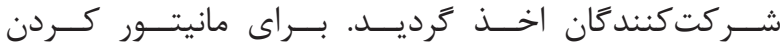

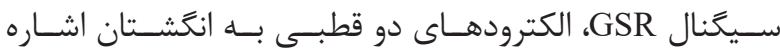

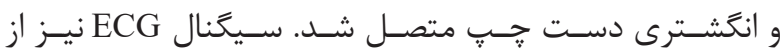

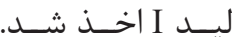

تمـــام ثبتهــــا در آزمايشـــاه علــوم اعصــاب محاســبـاتى در

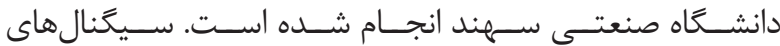
GSR و ECG 
خطـى ميــان دو گَروه نشــان داده شـده اسـت.

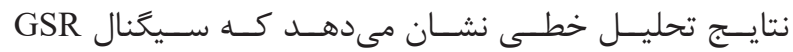

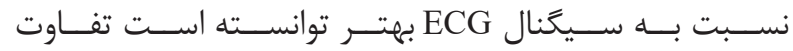

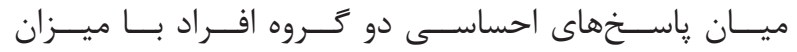

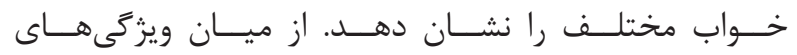

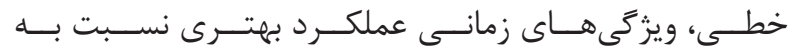

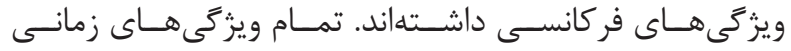

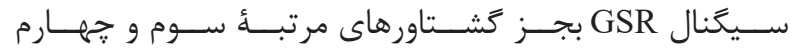

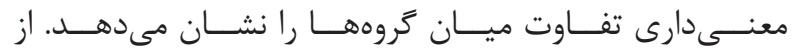

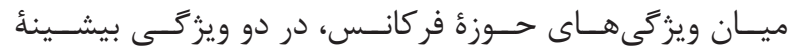

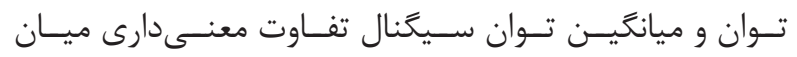

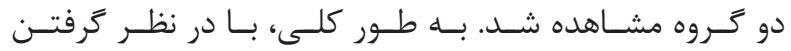

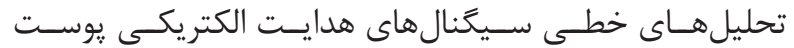

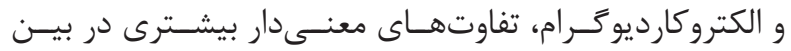

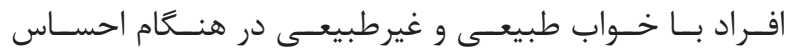

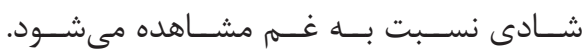

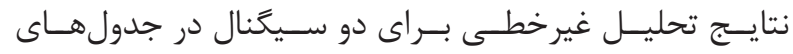

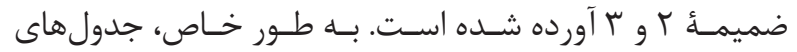

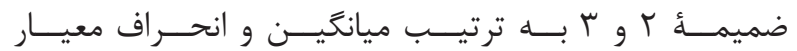

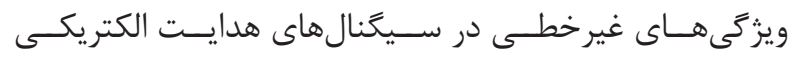

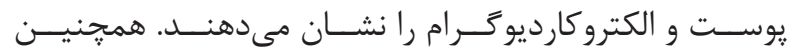

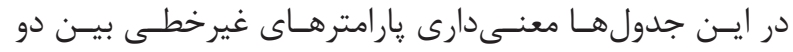

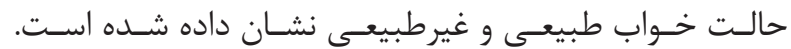

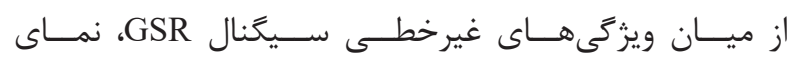

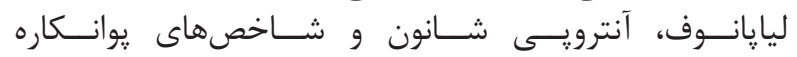

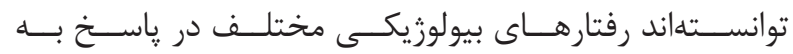

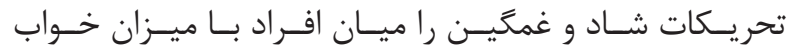

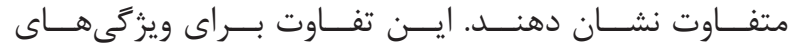

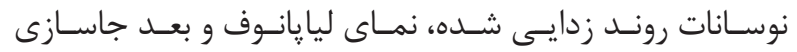

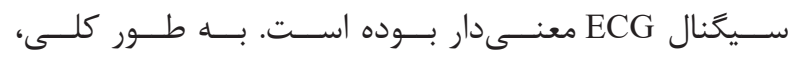

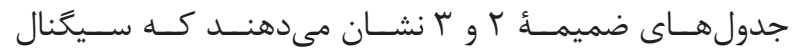

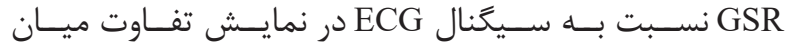

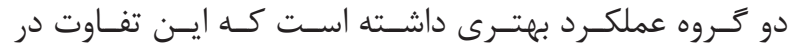

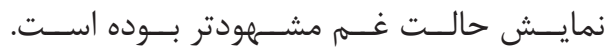

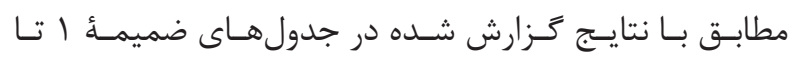

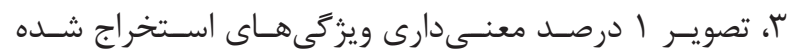

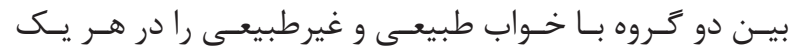

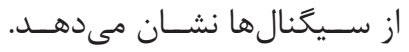

نتايـج بيانخــــ آن اسـت كـهـ بيشـترين تفــاوت معنسى دار بيـن

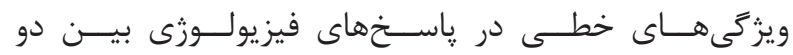

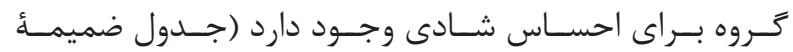

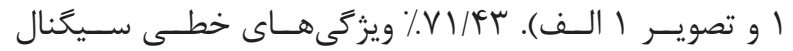

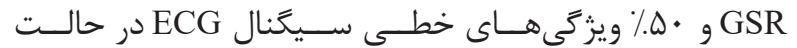

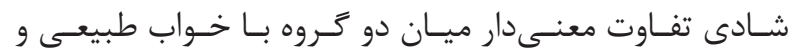

${ }^{12}$ Standard deviation of RR intervals

${ }^{13}$ Recurrence quantification analysis

${ }^{14}$ Recurrence rate

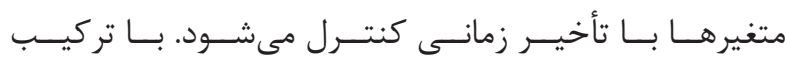

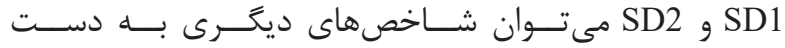

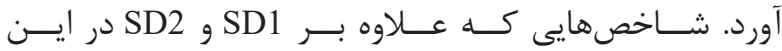

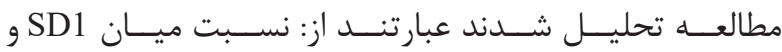
SD12) SD2

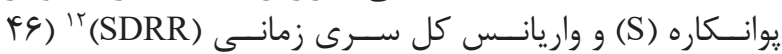

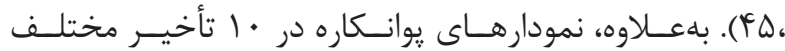

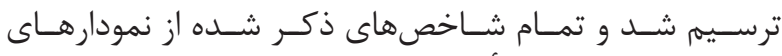

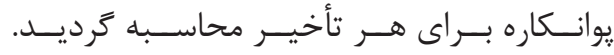
نمـاى ليايانـوف: مطابـق با روش ارائه شـده توســـ روزنســين

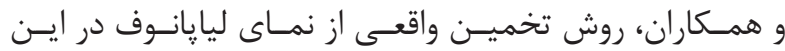
تحقيـق فراهـم كرديــ (FV)

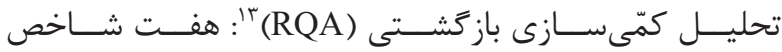

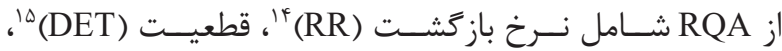

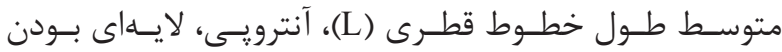
طال

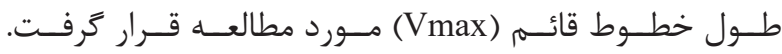

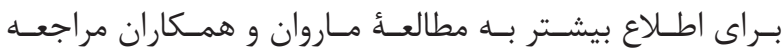

شـود (i) (1).

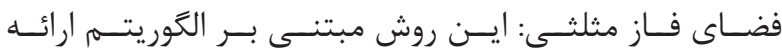

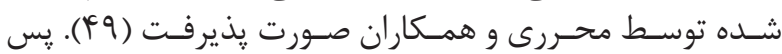

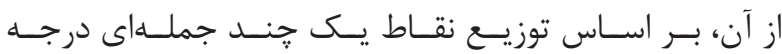

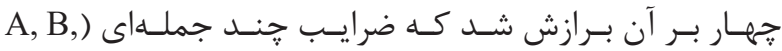

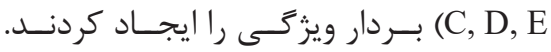

$$
\text { تحليل آمارى }
$$

در ايـن مطالعـه، بهمنظــور تحليـل تفــاوت ميــان رِاســخهاى

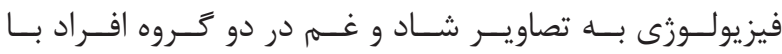

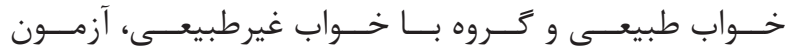

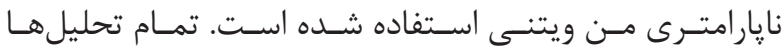

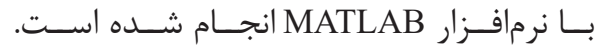

بافتهها

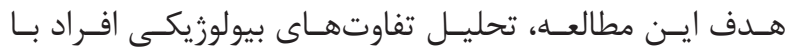

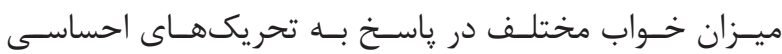

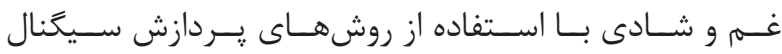

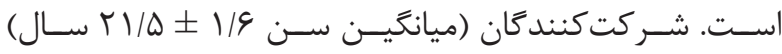

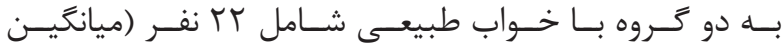

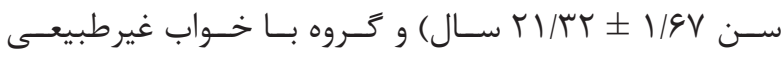

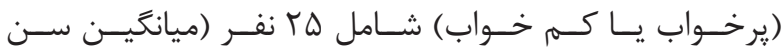

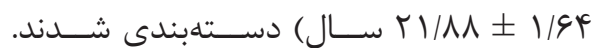

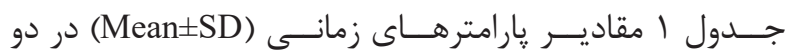

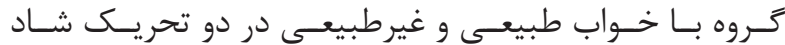

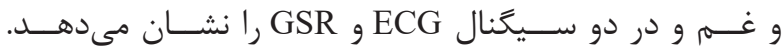

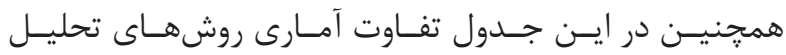

${ }^{15}$ Recurrence rate

${ }^{16}$ Laminarity 
مختلـف بــر موضـوع فعالســازى سيسـتم اعصـاب خودمختــار IV (ANS)

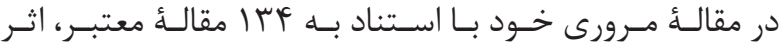

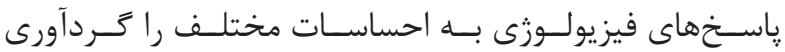

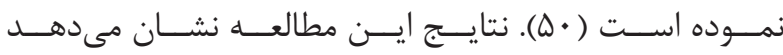

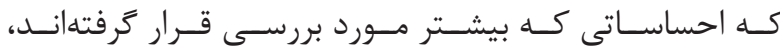

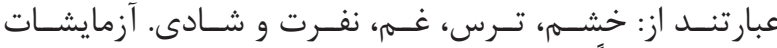

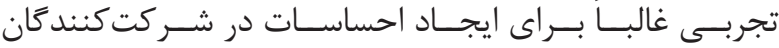

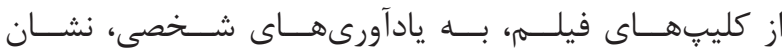

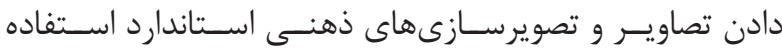

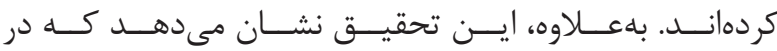

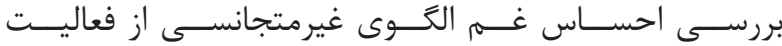

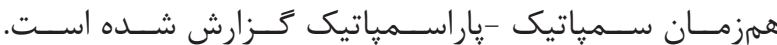

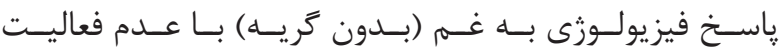

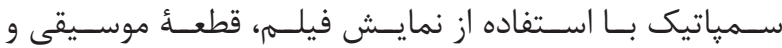

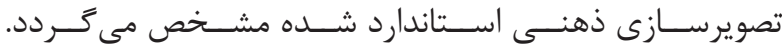

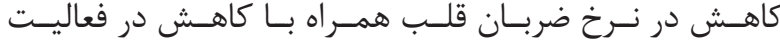

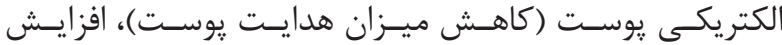

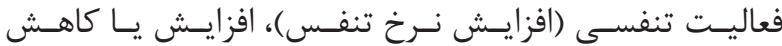

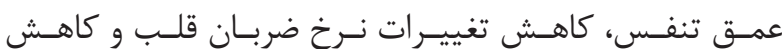

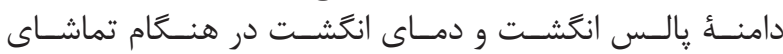

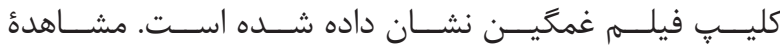

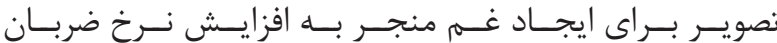

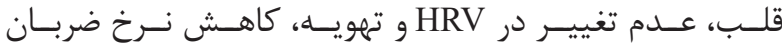

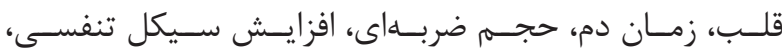

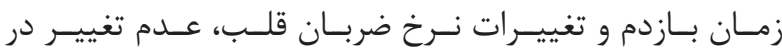

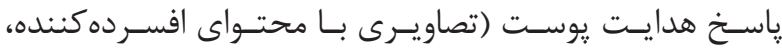

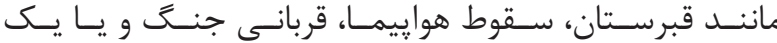

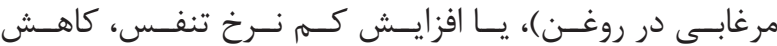

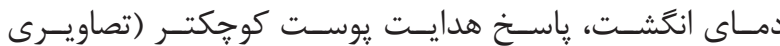

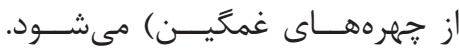

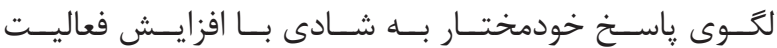

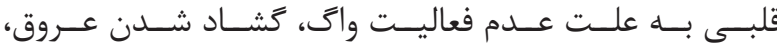

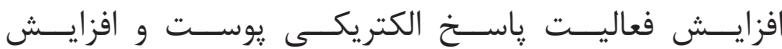

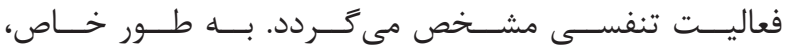

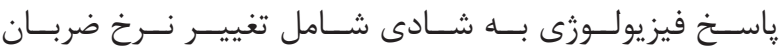

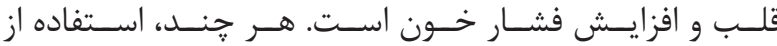

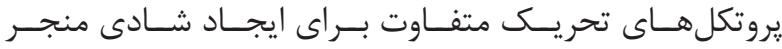

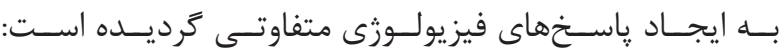

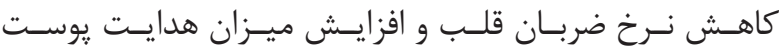

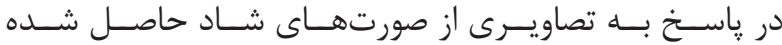

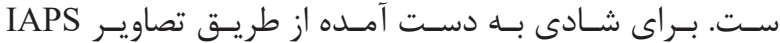

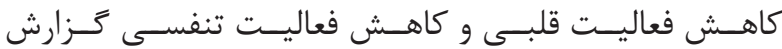

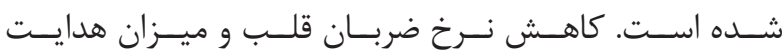

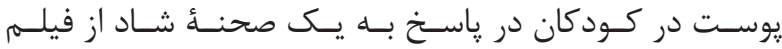

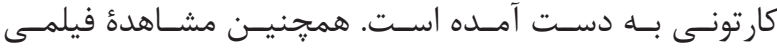

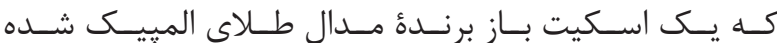
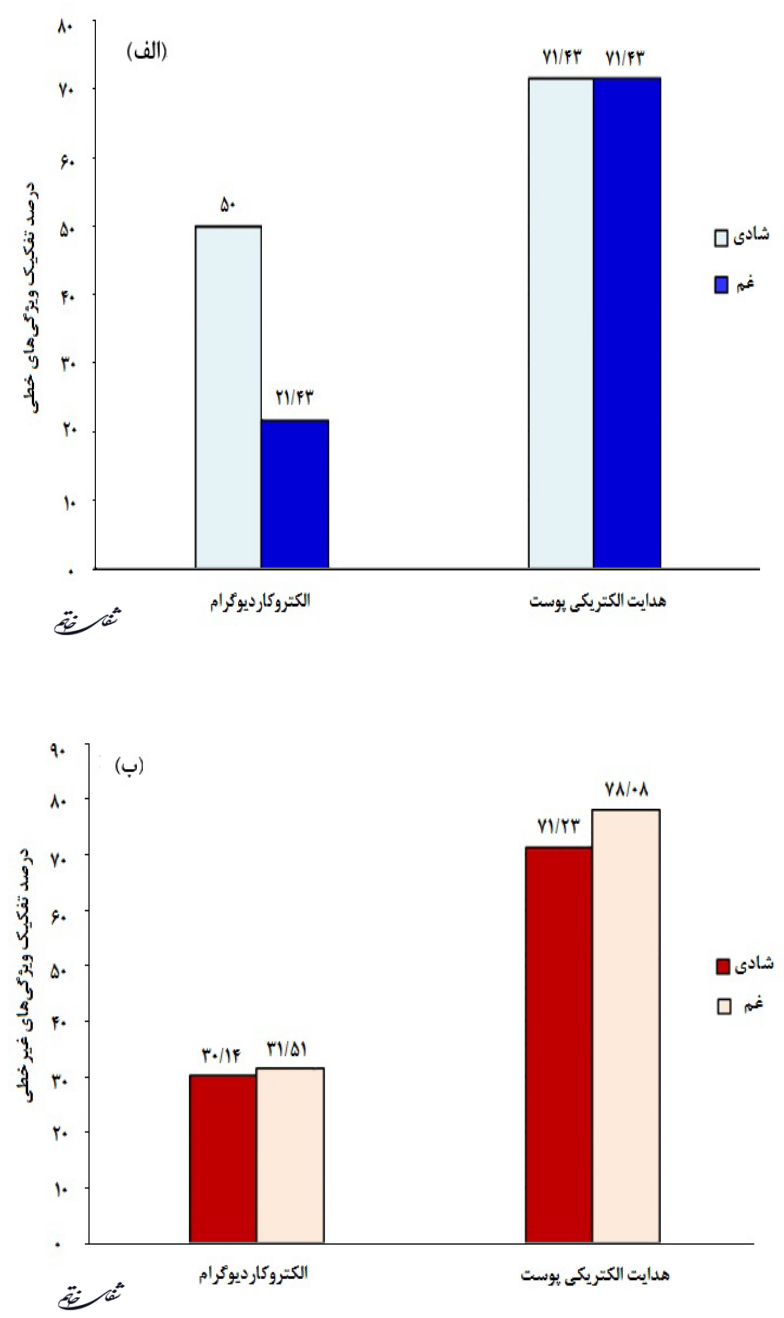

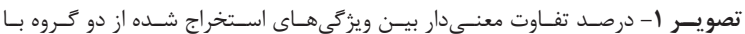

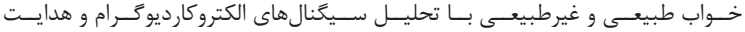

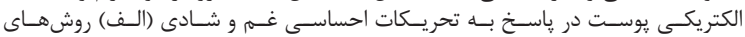

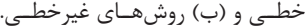

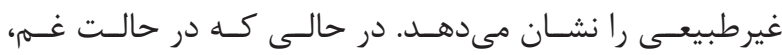

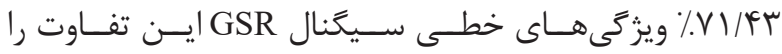

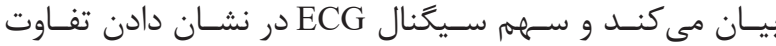

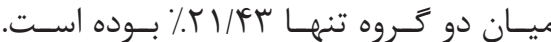

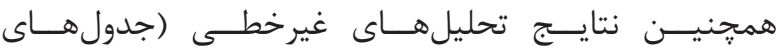

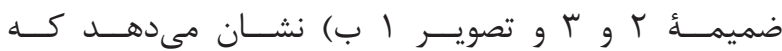

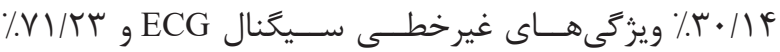

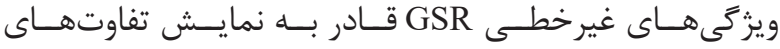

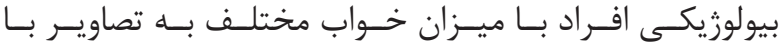

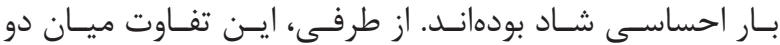

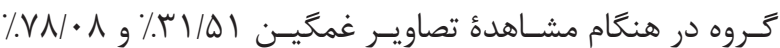

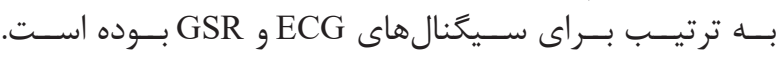
بحث و نتيجه كيرى

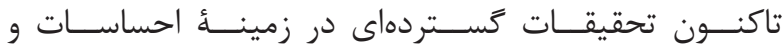

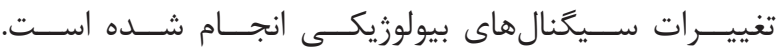

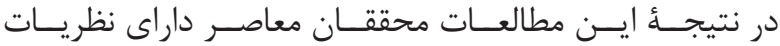




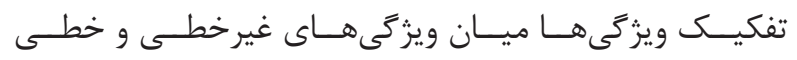

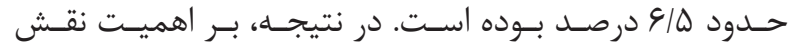

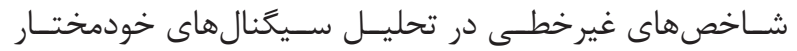

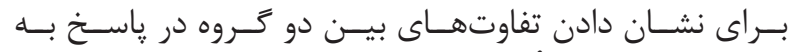

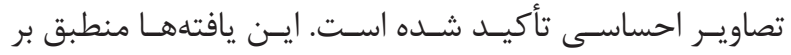

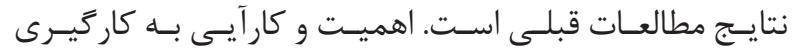

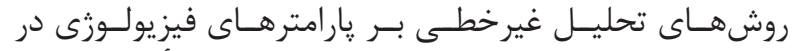

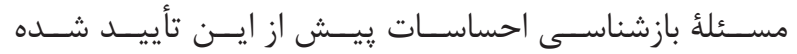

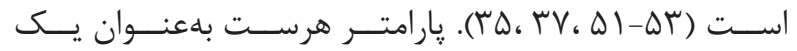

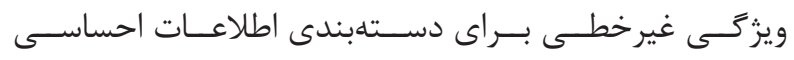

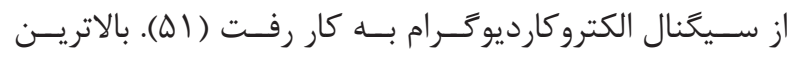

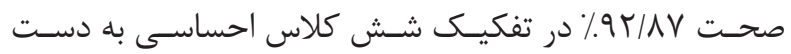

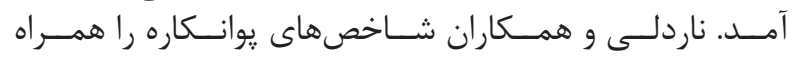

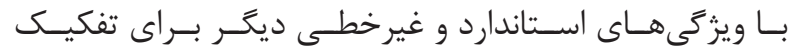

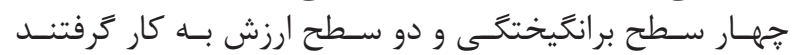

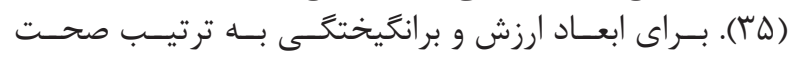

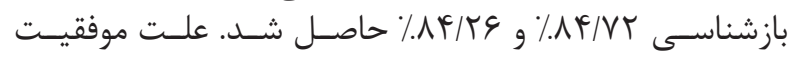

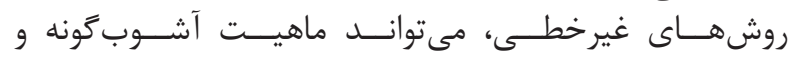

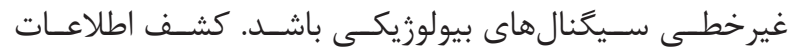

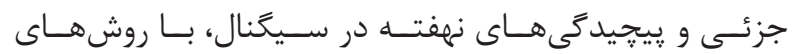

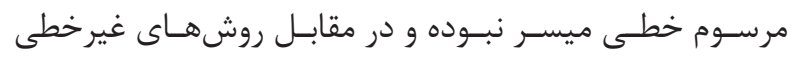

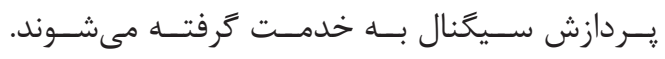

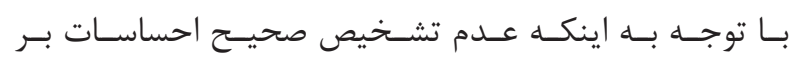

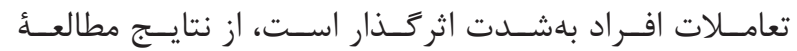

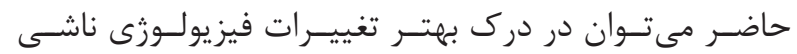

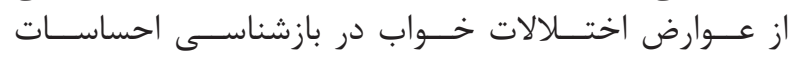

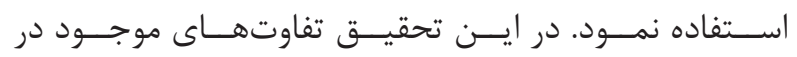

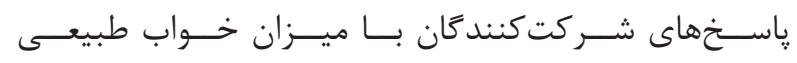

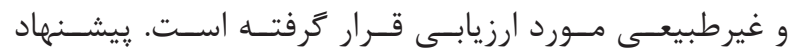

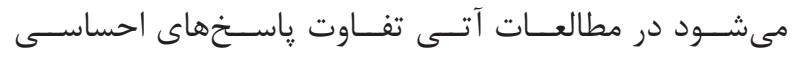
GSR و GCG

$$
\text { بررسـى قـرار كيــرد. }
$$

$$
\text { تشكر و قدردانى تمرسى }
$$

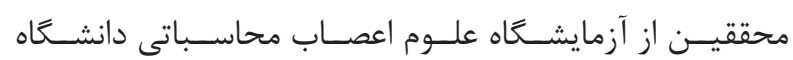

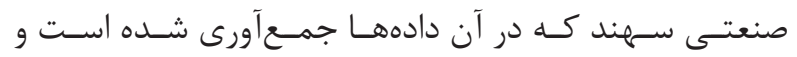

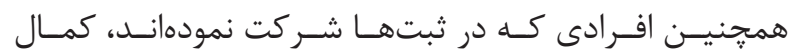

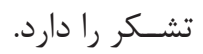

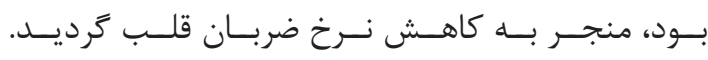

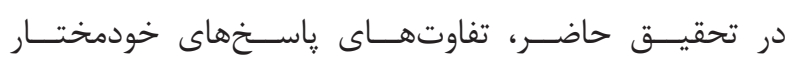

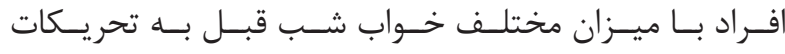

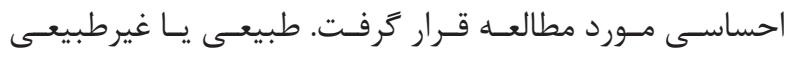

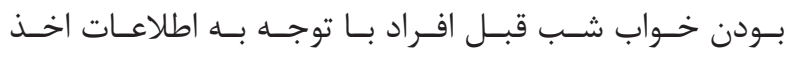

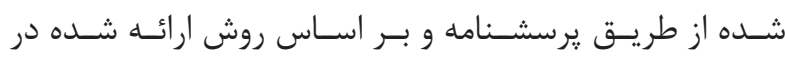

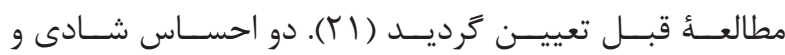

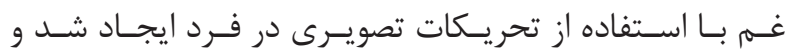

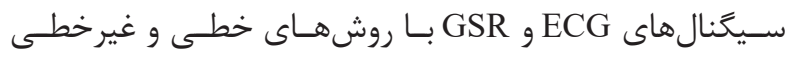

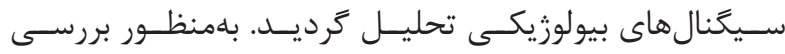

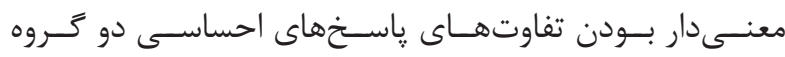

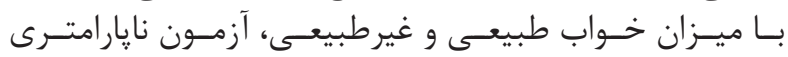

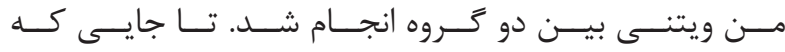

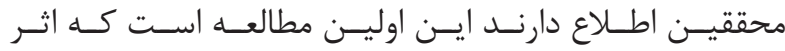

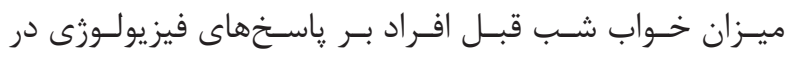

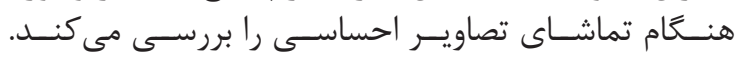

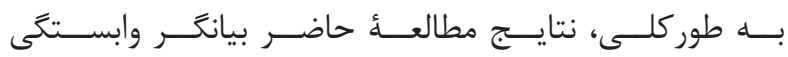

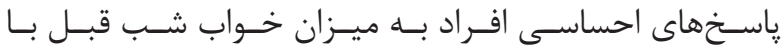

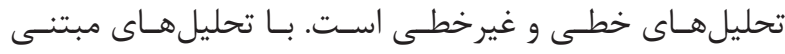

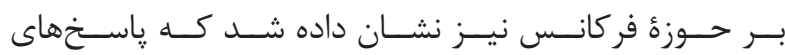

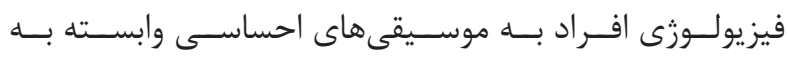

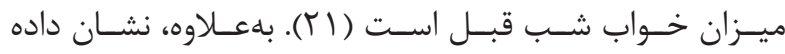

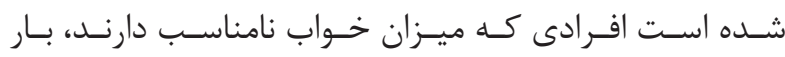

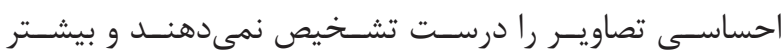

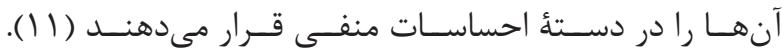

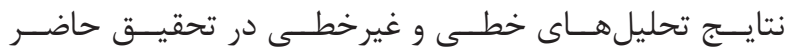

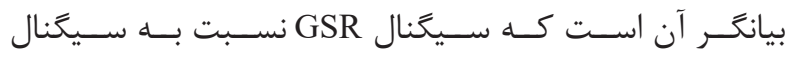

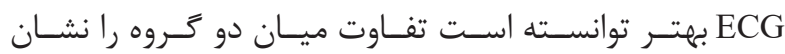

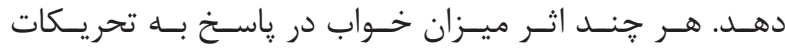

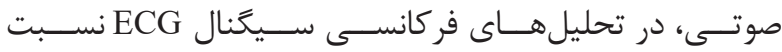

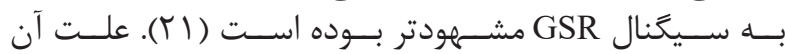

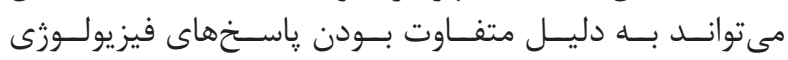

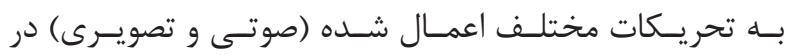

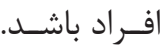

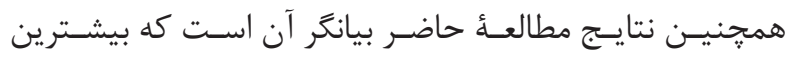

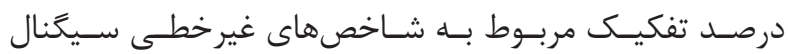

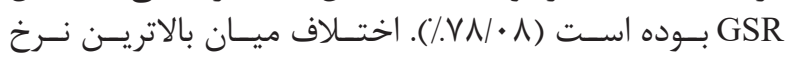




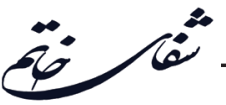

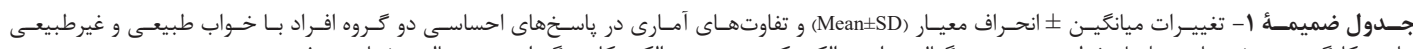

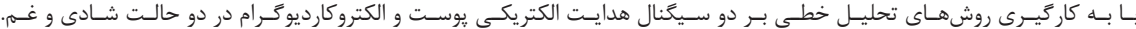

\begin{tabular}{|c|c|c|c|c|c|c|c|}
\hline & غم & & & شادى & & & \\
\hline $\mathbf{p}$ & خواب طبيعى & غيرطبيعى غواب & $\mathbf{p}$ & خواب طبيعى & خواب غيرطبيعى & ويرُكى & سيعْنال \\
\hline.$/ . r A r \%$ & $. / 1.9 \pm . / 1 r 9$ & $\cdot / \cdot \Delta r \pm \cdot / 1 \mathrm{rr}$ & $.1 \cdot 1 \cdot Y * \frac{\pi}{\pi}$ & ./Irrt.//Vr & $\cdot / 1 \cdot v \pm \cdot / 19 \mathrm{~V}$ & انحر اف معيار & \\
\hline 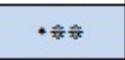 & $-\cdot / F V T \pm H / G V T$ & $-r /|\Lambda| \pm F / \Delta \Delta r$ & 숮쥬 & $-\cdot / F \Delta G \pm r / G \Delta F$ & $-r / \cdot \Delta G \pm r / \Delta G G$ & ميانكين & \\
\hline •슝슈 & $-\cdot / r V Q \pm r / V \Delta \Lambda$ & $-Y / \cdot Y \pm F / \Delta Q F$ & - 슉슈 & $-\cdot / r \mid \pm r / Y A \Lambda$ & $-1 / \wedge \Delta V \pm F / G \wedge F$ & بيشينه & \\
\hline - 수궂 & $-. / 9 F V \pm r / \Delta Q \mu$ & $-r / r r \mid \pm F / \Delta r$ & ·슈슈 & $-\bullet / G G F \pm r / \Delta G F$ & - r/TrTIF/FqT & كمينه & \\
\hline •슞추 & $-\cdot / F \vee q \pm r / G \wedge \Delta$ & $-r / 19 \Delta \pm F / \Delta G$ & 슈규 & $-\cdot / k q 9 \pm$ r/gr & $-r / \cdot V r \pm F / \Delta \Delta r$ & 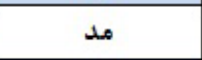 & \\
\hline 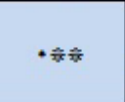 & $-\cdot / F V \Delta \pm r / G V F$ & $\begin{array}{c}r / 1 \Lambda F \pm F / \Delta \Delta F \\
-\end{array}$ & - 슈ㅈㅠㅠ & $\begin{array}{c}. / F 9 V \pm r / 9 F A \\
-\end{array}$ & $-r / \cdot q \Delta \pm F / \Delta \varphi$ & ميانه & 3 \\
\hline . & $\cdot|\cdot r \Lambda \pm \cdot| \cdot v \mid$ & $.1 .4 Y \pm .1 .94$ & $\cdot 1 \cdot 1 \cdot r$ 추 & $. / . * A \pm . / 1 \% G$ & $. / . r q \pm \cdot / 1 \% \Delta$ & واريانس & $\frac{3}{3}$ \\
\hline -ITKEA & $.1 .9 F \pm \cdot / \Delta r r$ & $\cdot|\cdot r g \pm \cdot / \Delta| \Lambda$ & .1 .941 & $. / 1 r v \pm \cdot / \Delta 9 \Lambda$ & $\cdot / \cdot \Delta \Delta \pm \cdot / \Delta$ & كشتاور سوم & $\overline{3}$ \\
\hline.$/ 914 F_{*} *$ & $r / r \cdot r \pm 1 /|\Delta|$ & $r / r \Lambda \Lambda \pm \cdot / \Lambda / 9$ & $.19 \wedge \mathrm{V}$ & $r / F \pm 1 / 1 \wedge r$ & $r / r \cdot \mid \pm \cdot / \Lambda 9 \Lambda$ & كشتاور جههارم & 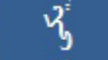 \\
\hline $.1 . \cdot r \Lambda \approx$ & $r / 9 \wedge F \pm r / 191$ & $r / 9 r r \pm r / 19 F$ & $.1 . Y F Y=$ & $r / 9 V V \pm r / / V 1$ & $r / \Lambda \Delta r \pm r / r$ & ريشه ميانكين & \\
\hline $.1 . \cdot r \Delta *$ & $\Delta / 9 \Delta \pm F / \%$ & V/AG & $.1 . r \mathrm{rV}$ 券 & $\Delta / Q T \Delta \pm F / r G$ & $\cdot / T \Delta V \pm G / F T$ & بيشينه توان & \\
\hline $.1 . \cdot r \Delta \leqslant$ & $\bullet / . \cdot v \pm \cdot / \cdot \bullet \Delta$ & $\bullet / * 9 \pm \cdot / * \wedge$ & $.1 . Y Y F=$ & $\bullet / \cdots v \pm * / \cdots \Delta$ & $\bullet / . \bullet \mid \pm \cdot / \cdot \bullet V$ & ميانكين توان & \\
\hline - /ArqF & $\cdot / . . r \pm \cdot / \cdot 1 r$ & $\cdot|\cdot \cdot| \pm \cdot|\cdot| \mid$ & ./1rq & $\cdot / \cdot \cdot 1 \pm \cdot / \cdot 1 \pi$ & $\cdot / \cdot * F \pm \cdot / \cdot 1 \Delta$ & فركانس بيشينه & \\
\hline - IATQF & $\cdot / \cdot . r \pm \cdot / \cdot 1 r$ & $\cdot \mid \cdot \cdot \pm \cdot / \cdot 14$ & ./Ir & $\cdot|\cdot \cdot| \pm \cdot / \cdot 1 F$ & $\cdot / \cdot . * \pm \cdot / \cdot 19$ & فركانس ميانكين & \\
\hline.$/ 9 Y \Delta A$ & $\cdot / 1 \cdot V \pm \cdot / \cdot F V$ &. $\mid 1 \cdot 9 \pm \cdot 1 \cdot 41$ & - IAFIA & $\cdot / 11 \pm \cdot / \cdot \Delta r$ & $\cdot \mid 1 \cdot v \pm \cdot 1 \cdot * 1$ & انحراف معيار & \\
\hline.$/ 9111$ & $-\cdot /|1| \pm \cdot / \cdot r q$ & $-\cdot / 1 V q \pm \cdot 1 \cdot F F$ &.$/ 1 F 9 F$ & $-\cdot / 1 V A \pm \cdot / \cdot F F$ & $-\cdot / 1 \vee q \pm \cdot 1 \cdot \vee \Delta$ & ميانكين & \\
\hline$\cdot / \Delta V \Delta q$ & ./HIF_./TAT & $\cdot /$ r/g士•/rVA & ./FTV & ./THFE./TAG & $. / H / 9 \pm \cdot / T V \Delta$ & بيشينه & \\
\hline$\cdot 1 \cdot \cdot \wedge r$ 추 & $-\cdot / k r q \pm \cdot / 1 r \Delta$ & $-\cdot / F 1 \Lambda \pm \cdot / 1 r q$ & $\cdot / \cdots \Delta *$ & $-\cdot / F \Delta F \pm \cdot / 19 \Delta$ & $-\cdot|F Y| \pm \cdot|| r \mid$ & كمينه & \\
\hline $.1 \cdot 11 \%$ & $-\cdot / T Y \pm \cdot / \cdot \Delta G$ & $-\cdot \mid r+1 \pm \cdot / \cdot \Delta$ & $.1 \cdot \Gamma Y_{\text {츄 }}$ & $-\cdot / T \mid V \pm \cdot / \cdot G$ & $-\cdot|r T| \pm \cdot|\cdot \Delta|$ & مد & \\
\hline$\cdot 1 \cdot \Delta \Delta$ & $-\cdot / r \cdot F \pm \cdot / \cdot F V$ & $-\cdot \cdot / r \cdot F \pm \cdot / \cdot F V$ & $\cdot 1 \cdot \cdot \Delta *$ & $-\cdot|r \pm \cdot| \cdot \Delta \mid$ & $-\cdot \cdot r \cdot r \pm \cdot / \cdot F \lambda$ & ميانه & \\
\hline.$/ 9 T \Delta A$ & $.1 .18 \pm . / .19$ & $. / \cdot 1 r \pm \cdot / \cdot 1$ & - IAFIA & $\cdot|\cdot| \Delta \pm \cdot|\cdot r|$ & $\cdot / \cdot 1 r \pm \cdot / \cdot 11$ & واريانس & \\
\hline . $/ 11 \mathrm{Tr}$ & $1 / T / F \pm 1 / F V V$ & $1 / \Delta 9 \Lambda \pm 1 / r q 1$ & $.1 . Y T F=$ & $\mid / T / \pm 1 / \Delta / \Delta$ & $1 / \Delta r F \pm 1 / r \cdot 9$ & كشتاور سوم & $\overline{3}$ \\
\hline $.1 .4 \wedge 9 \%$ & A/rATEF/GrV & $\Delta / 1 F 9 \pm \Delta / \cdot 9 \Delta$ & $-1 \cdot F \Delta 9 \%$ & A/TGY $\pm F / \Delta \Lambda T$ & $\Lambda / 1 \cdot \Delta \pm \Delta / 1 \neq q$ & كشتساور جههارم & 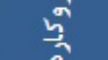 \\
\hline - ITYGF & $\cdot|r| r \pm \cdot / * F V$ & $\cdot|r| r \pm \cdot|\cdot F|$ & سזו/. & $\cdot|r| F \pm \cdot / \cdot \Delta F$ & . $/ \mathrm{r} \mid \mathrm{K} \pm \cdot / \cdot \mathrm{F}$ & ريشه ميانّين & $\frac{3}{2}$ \\
\hline.$/ 9194$ & •/rGY & $\cdot / r \Delta V \pm \cdot / \cdot \Lambda \Lambda$ &.$/ 1119$ & $\cdot / r \Delta \Lambda \pm * / \cdot \wedge q$ & $. / r \Delta V \pm . / \cdot 9$ & بيشينه توان & \\
\hline$\cdot / 1 \Lambda 9 V$ & $\begin{array}{c}-r \\
\cdot|\cdot \cdot| \pm r / V \times \mid\end{array}$ & $\begin{array}{c}-r \\
\cdot|\cdot \cdot| \pm r / 9 \times \mid\end{array}$ & ./TAIV & $\begin{array}{c}-r \\
\cdot|\cdot \cdot| \pm r|\Lambda \times|\end{array}$ & $\begin{array}{c}-r \\
\cdot|\cdot \cdot| \pm r / 9 \times \mid .\end{array}$ & ميانكين توان & \\
\hline - GATAr & $\begin{array}{l}-r \pm \cdot \mid \cdot \bullet \Delta \\
r /|V \times| \cdot\end{array}$ & $\cdot / \cdot \Delta \pm \cdot / \cdot \nabla F$ & .1 .199 * & $\cdot / \cdot \mid \pm \cdot / 11$ &. \pm & فركانس بيشينـه & \\
\hline .19 & $\begin{array}{l}-F \pm \cdot / . . q \\
r /|V \times|\end{array}$ & $\cdot / \cdot \cdot \Delta \pm \cdot / \cdot \nabla F$ & $.1 .199 \%$ & $\cdot 1 \cdot 1 \pm \cdot / 14$ & $\cdot \pm$ & فركانس ميانكين & \\
\hline
\end{tabular}




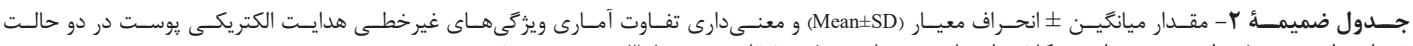

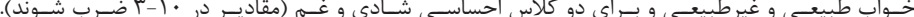

\begin{tabular}{|c|c|c|c|c|c|c|c|c|}
\hline$P$ & خواب غيرطبيعى & خواب طبيعى & & $P$ & خواب غيرطبيعى & خواب طبيعى & & \\
\hline \multicolumn{3}{|c|}{ SD2 } & ل إتأخير & \multicolumn{3}{|c|}{ SD1 } & 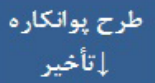 & \\
\hline * & $\mid Q 1 / A 9 \pm T r \Delta / F$ & $\mid A G / F A \pm T F Q / T$ & 1 & Ns & $1 / Y r \pm r Y / r A$ & $1 / 9 q \pm r 1 / 9 p$ & 1 & \\
\hline * & $|Q| / A T \pm T r \Delta / P$ & IAG/FGETFQ/T & r & Ns & $r / / q \pm \Delta \Delta / Q T$ & $r / / F \pm r A / / V$ & r & \\
\hline * & |Q1/ATERTA/Q & IAG/FADTFA/T & $r$ & Ns & $r / F Y \pm V \Delta / T q$ & $r / F / \pm \Delta r / 9 \Delta$ & $r$ & \\
\hline$*$ & $|\Delta| / \Lambda \mid \pm r r \Delta / \Delta$ & IAG/FPETFQ/T & f & Ns & $r / 9 V \pm q v / r r$ & $r / 9 r \pm 99 / \cdot r$ & f & \\
\hline * & $101 / 1 \Delta \pm r \Delta \Delta / \Delta$ & MAG/FFEYFQ/ & $\Delta$ & Ns & $r / \Lambda T \pm I / r$ & $r / \vee Q \pm \Lambda G / \Lambda F$ & $\Delta$ & \\
\hline * & $\mid \Delta 1 / \Lambda \pm r r \Delta / \theta$ & $1 \Lambda G / F T \pm r F Q / T$ & 9 & Ns & $r / 9 \gamma \pm 1 / p$ & $r / 9 r \pm 1 / 1$ & 9 & \\
\hline * & $|\Delta| / \Lambda \pm r r \Delta / \Delta$ & MAG/FTETFQ/ & $\mathrm{V}$ & Ns & $r / \cdot 9 \pm 1 / v$ & $r / \cdot \Delta \pm 1 / r$ & $\mathrm{v}$ & \\
\hline * & $|Q| / A \pm r r \Delta / P$ & MAG/PTEYFQ/T & $\wedge$ & * & $r / 1 r \pm r$ & $r / / F \pm 1 / \Delta$ & $\wedge$ & \\
\hline * & 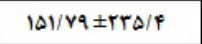 & MAG/PTETFQ/T & 9 & Ns & $r / T r \pm r / T$ & $r / \cdot r \pm 1 / r$ & 9 & \\
\hline * & $101 / v 9 \pm r r \Delta / F$ & IAG/FTEYFQ/T & 1. & Ns & $r / r F \pm r / \theta$ & $r / f F \pm 1 / 9$ & 1. & \\
\hline \multicolumn{3}{|c|}{ Area } & & \multicolumn{3}{|c|}{ SD1/ SD2 } & & \\
\hline * & $. / 9 \vee \pm r$ & $1 / 1 r \pm 1 / 1$ & 1 & * & $p q / r q \pm \Delta F / p$ & $p+/ \Delta \pm F v / r$ & 1 & \\
\hline * & $1 / r \pm r$ & $1 / F \wedge \pm r / \Delta$ & $r$ & * & $91 / 9 \Delta \pm 9 \mathrm{~V} / \mathrm{A}$ & $\Delta \cdot / \Gamma \Delta \pm \Delta \Lambda / 9$ & $r$ & \\
\hline * & $1 / \Delta \varphi \pm p$ & I/VF $\pm r$ & $r$ & * & GN/TA $\pm Y r / \Delta$ & $\Delta \Delta / \wedge 9 \pm 9 \Delta / 1$ & $r$ & \\
\hline * & $1 / \wedge \pm \Delta / 1$ & $1 / 9 \wedge \pm r / V$ & p & * & $\mathrm{V} / \mathrm{AV} \pm \mathrm{V} 9 / 9$ & $\Delta 9 / 91 \pm 99 / 1$ & p & \\
\hline * & $r / . r \pm 9 / T$ & $r / T \pm F / r$ & $\Delta$ & * & $\mathrm{VF} / \mathrm{QT} \pm \mathrm{VQ/Q} / \mathrm{Q}$ & $G T / / T \pm Y r / Y$ & $\Delta$ & \\
\hline * & $T / T F \pm V / T$ & $T / F T \pm F / Q$ & 9 & * & $\mathrm{V} / \mathrm{V}| \pm \wedge|$ & $G F / / T \pm V \Delta / 1$ & 9 & \\
\hline * & $r / F \Delta \pm \Lambda / F$ & $r / 9 r \pm \Delta / 9$ & $\mathrm{v}$ & * & $V q / p \pm V q / f$ & $9 \% / Q \Lambda \pm V \Delta / Q$ & $\mathrm{v}$ & \\
\hline * & $r / 99 \pm 9 / \Delta$ & $r / \wedge r \pm 9 / r$ & $\wedge$ & * & $V \Delta / r q \pm V V$ & 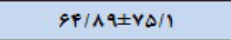 & $\wedge$ & \\
\hline * & $r / A Y \pm 1+/ 9$ & $\mathrm{r} / \cdot \mathrm{v} \pm \mathrm{r}$ & 9 & * & $\mathrm{YA} / \cdot \mathrm{I} \pm \mathrm{A} \cdot / 1$ & $99 / \mathrm{V} \Delta \pm \mathrm{V} 9 / 9$ & 9 & \\
\hline * & $r / 1 \pm 11 / \checkmark$ & $r / r q \pm v / r$ & 1. & * & $\mathrm{VQ/qV} \pm \mathrm{Vq} / \mathrm{f}$ & SV/A $9 \pm V Y / V$ & 1. & \\
\hline \multicolumn{3}{|c|}{ RQA } & ل إانديس & \multicolumn{3}{|c|}{ SDXX } & & \\
\hline * & $9 / 9 \pm T / T$ & $v \pm r / \Delta$ & RR & * & $1+V / P 1 \pm 199 / 0$ & $|r| / \wedge \wedge \pm \mid \vee r / P$ & 1 & \\
\hline Ns & $|v / v \pm F /|$ & $11 / 1 \pm p / T$ & قطعيت & * & $1+V / F \mid \pm 199 / 0$ & $|r| / A \wedge \pm \mid V T / F$ & $r$ & \\
\hline Ns & $11 v / r \pm 1 v / 9$ & $|r| / \Lambda \pm T \Lambda / F$ & $\mathbf{L}$ & * & $1-V / P| \pm| 99 / 0$ & $|r| / \wedge \wedge \pm \mid \vee r / F$ & $r$ & \\
\hline Ns & rIg/VIIfI & $r F V / r \pm r / 9 / 9$ & $L \max$ & * & $1-V / F / \pm 199 / 0$ & $|r| / \wedge \wedge \pm \mid V T / F$ & f & \\
\hline * & $\mathrm{Fr} / \mathrm{V} \pm 11 / \mathrm{r}$ & $P \Delta / \Delta \pm I T / G$ & آنترويى & * & $1-V / F 1 \pm 199 / 0$ & $|r| / \wedge \wedge \pm \mid V T / F$ & $\Delta$ & \\
\hline Ns & $10 / 9 \pm 9 / 4$ & $10 / Y \pm 9 / 1$ & LAM & * & $1-V / F 1 \pm 199 / 0$ & IrV/AA \pm IVY/F & 9 & \\
\hline Ns & $r \mid F / T \pm \| 1 / T$ & rTVEIrq/T & $V \max$ & * & $1+V / F 1 \pm 199 / 0$ & $|r| / A \wedge \pm \mid V T / F$ & $\mathrm{v}$ & \\
\hline * & $19 r \pm F 910$ & $109 / f \pm r q$ & T1 & * & $1-V / P 1 \pm 199 / 0$ & $|r| / \wedge \wedge \pm \mid V T / F$ & $\wedge$ & \\
\hline \multirow[t]{2}{*}{ Ns } & $r q \pm=/ \Delta$ & $r q \pm=/ p \wedge$ & Trans & * & $1+Y / F 1 \pm 199 / 0$ & $|r| / \wedge \wedge \pm \mid V T / F$ & 9 & \\
\hline & & & & * & $1 . Y / P 1 \pm 199 / 0$ & $|r| / A \wedge \pm \mid V T / P$ & 1. & \\
\hline \multicolumn{3}{|c|}{ ضرايب خندجملهاى } & ل إانديس & & & & لآنتروجى & \\
\hline Ns & $-r T / \varphi \pm \Delta \Delta T / \Lambda$ & $-r / 1 \pm 9 T / 9$ & A & Ns & $-|\digamma A / V \pm| r \Delta / T$ & $-1 \Delta r / 9 \pm 1 r \Delta$ & تخمين & \\
\hline Ns & $-T / T \pm F F g / T$ & r/g士rri/r & B & * & $-r|\cdot r \times| 0^{-\lambda} \pm g|r| x||^{-1}$ & $-1 / v 9 \times 10^{+\lambda} \pm r / r 9 \times 10^{-1}$ & شانون & \\
\hline Ns & $q \cdot \Delta / A \pm F \Delta \xi / F$ & $q Q 1 / Y \pm F F \wedge / T$ & C & * & $q / 9|\times|+^{-4} \pm q / \cdot V \times \mid 0^{-4}$ & $\Delta / 9 \times 1+^{-4} \pm 1 / T \times 1+^{-8}$ & لثاريتمى انرثى & \\
\hline$* *$ & $f \times 1 *^{-f} \pm+\mid 9 \times 1 *^{-f}$ & $V T \Delta / 1 \pm V /|\Delta x| *^{-F}$ & D & * & $\Delta T / G \pm P V / A$ & $P I / \Delta \pm r \Delta / r$ & نمونه & \\
\hline * & $r / F v_{x}\left|+^{-\Delta} \pm r\right| \Delta x \mid+^{-\Delta}$ & $1 / r r \times\left.\right|^{-\Delta} \pm 1 /\left.\wedge \Delta x\right|^{-\Delta}$ & $\mathbf{E}$ & & & & & \\
\hline \multicolumn{3}{|c|}{ ضر ايب DFA } & ل ل ل لانديس & * & $\mathrm{rr} / \mathrm{A} \pm \mid \mathrm{V} / \mathrm{V}$ & 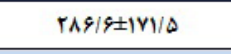 & نماى لياهانوف & \\
\hline * & $+/ \mid f \times 1 *^{-F} \pm r q 1 / r$ & +||$r \times \mid *^{+F} \pm r+\Lambda / \Delta$ & D & Ns & $P 9 q / \Delta \pm r+1 / 9$ & $F V F / P \pm T F q / F$ & يبيجيدى & \\
\hline * & $* /\left|f \times 1 *^{* F} \pm r q\right| / r$ & $-/|\gamma \times x|+{ }^{*} \pm r+1 / \Delta$ & $\alpha$ & Ns & $|x| 0^{-4} \pm \Delta r / \Delta$ & $\left.|x|\right|^{-4} \pm$ & بعد جاسازى & \\
\hline \multicolumn{3}{|c|}{ SD2 } & ل إأخير & \multicolumn{3}{|c|}{ SD1 } & طرح يوأكاره & \\
\hline * & $11 / \Lambda \pm \mid r r / F$ & $10 * / T \pm 1 A T / T$ & 1 & * & $1 / v \pm=/ r$ & $1 / v \pm * / \pi r$ & 1 & \\
\hline * & $11 / v q \pm \mid v r / f$ & $10 \cdot / T \pm 1 \Lambda T / T$ & r & * & $r / 1 \pm \cdot / F r$ & $r / 1 \pm \cdot / r p$ & $r$ & \\
\hline * & $11 / v q \pm \mid r r / p$ & $10 \cdot / T \pm 1 \Lambda T / T$ & $r$ & * & $r / P \pm=/ \Delta F$ & $r / P \pm \cdot / F r$ & $r$ & \\
\hline * & $11 / v q \pm \mid r r / p$ & $10 \cdot / T \pm 1 \Lambda T / T$ & f & * & $r / 0 \pm+191$ & $r|9 \pm+| \Delta \mid$ & f & \\
\hline * & $11 / v q \pm \mid r r / p$ & $10 \cdot / r \pm 1 A T / T$ & $\Delta$ & * & $r / v \pm \cdot / A r$ & $r / \gamma \pm+/ \Delta Q$ & $\Delta$ & \\
\hline$*$ & II/Vq & $10+/ T \pm 1 \Lambda T / r$ & 9 & * & r/A土*/99 & $r / 1 \pm+191$ & 9 & \\
\hline * & $11 / \vee q \pm \mid r r / p$ & $10 \cdot / T \pm 1 \Lambda T / T$ & V & $*$ & $r / \Lambda \pm 1 / r$ & r/Q土*/Vq & V & \\
\hline$*$ & $11 / v q \pm \mid r r / p$ & $10-/ T \pm 1 \Lambda T / T$ & $\Lambda$ & * & $r / q \pm 1 / r$ & r/q土-/9) & $\Lambda$ & \\
\hline * & $11 / v q \pm \mid r r / p$ & $10 \cdot / T \pm 1 A T / T$ & 9 & * & $r \pm 1 / \Delta$ & $r \pm 1$ & 9 & \\
\hline
\end{tabular}




\begin{tabular}{|c|c|c|c|c|c|c|c|c|}
\hline 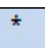 & $11 / \mathrm{Vq} \pm \mid \mathrm{Vr} / \mathrm{f}$ & $|Q+/ T \pm| A T / T$ & 1. & * & $r /| \pm| / \gamma$ & $r /| \pm| / r$ & 1. & \\
\hline \multicolumn{3}{|c|}{ Area } & & \multicolumn{3}{|c|}{ SD1/ SD2 } & & \\
\hline * & $. / 99 \pm 1 / r$ & $\cdot|\Lambda \Delta \pm| / r$ & 1 & * & $p v / \Delta \pm p q / r$ & $\rho \Delta / \Gamma \pm \Delta \mid / q$ & 1 & \\
\hline * & $+/ 9 \pm 1 / 9$ & $1 / 1 \pm 1 / 0$ & $r$ & * & $\Delta q / F \pm q 1 / r$ & $\Delta G / F \pm G F / q$ & $r$ & \\
\hline * & $1 / 1 \pm r / p$ & $1 / r \pm 1 / \Lambda$ & $r$ & * & $9 \Delta / r \pm 9 \Delta / A$ & $G r / \Delta \pm V / / \Lambda$ & $r$ & c \\
\hline * & $1 / r \pm r$ & $1 / p \pm r / 1$ & f & * & $9 N / 9 \pm 9 N / D$ & $99 / 9 \pm V 9 / 0$ & f & \\
\hline * & $1 / r \pm r / 9$ & $1 / \Delta \pm r / r$ & $\Delta$ & * & $Y / / q \pm V Y / q$ & $99 / \Upsilon \pm \mathrm{Yq} / \mathrm{q}$ & $\Delta$ & \\
\hline * & $1 / p \pm p / r$ & $1 / 9 \pm r / 9$ & 9 & * & $V r / q \pm V r / Y$ & $Y / / r \pm \Lambda r / F$ & 9 & \\
\hline * & $1 / \Delta \pm \Lambda$ & $1 / r \pm r / 9$ & v & * & $V Y / \Lambda \pm Y Y / Y$ & $Y / / q \pm \Lambda T / q$ & V & \\
\hline * & $1 / 9 \pm \Delta / \%$ & $1 / \Lambda \pm r / 1$ & $\wedge$ & * & $V r / 9 \pm \gamma \cdot / p$ & $V / / r \pm \Lambda 1 / \Delta$ & $\Lambda$ & \\
\hline * & $1 / Y \pm \varphi$ & $1 / 9 \pm r / p$ & 9 & * & $V \Delta / F \pm V Y / q$ & $r r \pm \Lambda r / l$ & 9 & \\
\hline * & $1 / A \pm 9 / 9$ & $\Gamma \pm \Gamma / \gamma$ & 1. & * & $Y Y / F \pm Y Q / Y$ & $V \varphi / / \pm \Lambda r / q$ & 1. & \\
\hline \multicolumn{3}{|c|}{$\mathrm{RQA}$} & لإنديس & \multicolumn{3}{|c|}{ SDXX } & & \\
\hline Ns & $r \varphi / v \pm r / p$ & $r F / v \pm r / \Delta$ & $\mathbf{R R}$ & * & $\Lambda r / p \pm \mid r r / 9$ & $1+9 / r \pm 1 \% \Lambda / 9$ & 1 & \\
\hline Ns & $r q / \Delta \pm+\Delta T$ & $\mathrm{rq} / \mathrm{st} \cdot \mathrm{pF}$ & قطعيت & * & $\Delta r / F \pm \mid r T / G$ & $1.9 / r \pm 1 \% \Lambda / 9$ & r & \\
\hline Ns & $Y \Delta G / \beta \pm \mid Y r / q$ & $V Y+|\Lambda \pm| V F / Y$ & L & * & $\Delta r / F \pm \mid r r / \varphi$ & $1.9 / r \pm 1 \% \Lambda / 9$ & $r$ & \\
\hline Ns & $19 \mathrm{Yr} / 1 \pm \mathrm{TP} / /$ & 199A//ATrA/A & $\operatorname{Lmax}$ & * & $\Lambda T / F \pm I r r / q$ & $1.9 / \% \pm 1 \% \Lambda / 9$ & f & \\
\hline Ns & $|r r / r \pm| r / r$ & $|r e / \Delta \pm| r / r$ & آنترويى & * & $\Lambda r / F \pm \mid r r / q$ & $1.9 / r \pm 1 \% \Lambda / 9$ & $\Delta$ & \\
\hline Ns & $r q / q \pm+/ r \Delta$ & $\mathrm{rq} / \mathrm{q} \pm+/ \mathrm{rr}$ & LAM & * & $\Delta r / \varphi \pm \mid r r / 9$ & $1+9 / r \pm 1 \% / / 9$ & 9 & \\
\hline Ns & $|g| Y / \Delta \pm r \mid Y / A$ & $|9 r+ \pm r+1| r$ & $V \max$ & * & $\Delta r / \& \pm \mid r r / G$ & $1+9 / r \pm 1 \% N / q$ & V & \\
\hline Ns & $P Q / Y \pm T / Y$ & $p \varphi / Y \pm r / A$ & T1 & * & $\Delta r / \beta \pm \mid r r / \varphi$ & $1+\xi / r \pm 1 \% N / q$ & $\Lambda$ & \\
\hline \multirow[t]{2}{*}{ Ns } & $\Gamma q / / \pm+/ T \Delta$ & $\mu q / / \pm+190$ & Trans & * & $\Delta r / \beta \pm \mid r r / 9$ & $1+9 / r \pm 1 \% N / 9$ & 9 & \\
\hline & & & & $*$ & $A r / \beta \pm \mid r T / \varphi$ & $1+9 / r \pm 1 \% N / 9$ & 1. & \\
\hline \multicolumn{3}{|c|}{ ضرايب جخناجملهلى } & ل إنديس & & & & لآنتروبى & \\
\hline Ns & $19 \% / 9 \pm+|p r x| *^{*}$ & $\Delta Y / Q \pm A Y A / I$ & A & Ns & $-|\Delta P / \Lambda \pm| r \Delta / \varphi$ & $-|9 \% / \Delta \pm| \mid \alpha / A$ & تخمين & \\
\hline * & $-|r e /| \psi \pm \cdot\left|\mu_{x}\right| *^{+4}$ & $-r|f / q \pm+| r \xi x \mid+^{+4}$ & B & * & $-p|+\Delta x|+^{-\Lambda} \pm g|y x| *^{-\Lambda}$ & -||$A x\left|*^{+\Lambda} \pm r\right| A \Lambda x \mid *^{+\Lambda}$ & شانون & \\
\hline Ns & $99 \% / \Delta \pm+\left.|| x\right|^{+4}$ & $\cdot|| x\left|*^{+F} \pm+\right| \psi A x \mid *^{+f}$ & C & * & $V / \Psi A x\left|*^{-4} \pm \Lambda / 9 \Psi x\right| *^{+4}$ & 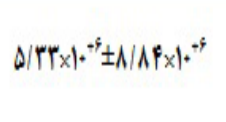 & لكاريتمى أرزىى & \\
\hline$* *$ & $+|f x|+{ }^{+F} \pm+|q| x \mid *^{+f}$ & $9 V / \Lambda \pm+|\Lambda 9 x|+*^{*}$ & D & Ns & $\Delta / / Y \pm p \mid$ & $p Y / Y \pm p i \mid q$ & نمونه & \\
\hline$i \pi$ & $r|\Delta x|^{+\Delta} \pm r /\left.p x\right|^{+\Delta}$ & $|/| r x|+\Delta \pm 1 / \Delta 9 x|+\Delta$ & E & & & & & \\
\hline \multicolumn{3}{|c|}{ ضر ايب. } & ل إنديس & $\dot{*}$ & $\operatorname{ror} / 9 \pm 199 / 1$ & $r+p / r \pm \mid r r / r$ & نماي لياهائوف & \\
\hline Ns & $+|f x|+{ }^{+*} \pm r \lambda y$ & 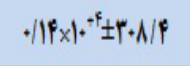 & D & * & erv/rtury/l & $\operatorname{per} / q \pm \mid \lambda \mathrm{r} / \mathrm{Y}$ & بيجيدكى & \\
\hline Ns & +|| $9 x \mid *^{+*} \pm r A y$ & $\cdot|19 x|+{ }^{+f} \pm r+1 / p$ & $\alpha$ & Ns & $1 \ldots \pm$ & $1 \ldots+\ldots$ & بعل جاسازي & \\
\hline
\end{tabular}




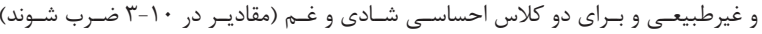

\begin{tabular}{|c|c|c|c|c|c|c|c|c|}
\hline$P$ & خواب غيرطبيعى & خواب طبيعى & & $P$ & خواب غيرطبيعى & خواب طبيعى & & \\
\hline \multicolumn{3}{|c|}{ SD2 } & لأخير & \multicolumn{3}{|c|}{ SD1 } & طرح بوانكاره & \\
\hline Ns & $1 \Delta \cdot / T \pm \Delta \Lambda / T$ & $1 \Delta F / \Delta \pm V F / \Lambda$ & 1 & * & $19 \pm \Delta / 1$ & $1 \Lambda / r \pm r / \gamma$ & 1 & \\
\hline Ns & $\mid f q / p \pm \Delta V / r$ & $1 \Delta r / A \pm V F / \Delta$ & r & * & $r F / / \pm 1 T / F$ & $\mathrm{rr} / \mathrm{T} \pm \mathrm{V} / \mathrm{T}$ & r & \\
\hline Ns & $\mid F 9 / \Delta \pm \Delta 9 / 9$ & $101 / 1 \pm V P / Q$ & r & * & $r V / F \pm 19 / 9$ & $r g / T \pm 1-1 T$ & r & \\
\hline Ns & $\mid F F / F \pm \Delta \Delta / r$ & $|p q \pm \gamma p /|$ & f & * & $F F / r \pm r / / Q$ & Fr/G士F/l & f & \\
\hline Ns & $\mid F-/ Y \pm \Delta F / \Delta$ & $\mid f \Delta / r \pm V r / q$ & $\Delta$ & * & $\Delta P / \Delta \pm T F$ & $\Delta F / T \pm I V$ & $\Delta$ & \\
\hline Ns & $\mid r \Lambda / r \pm \Delta r / r$ & $\mid F T / \Delta \pm V Y / P$ & 9 & * & 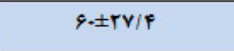 & $9+19 \pm r+19$ & 9 & \\
\hline Ns & $\mid T F / \Delta \pm \Delta r / \Delta$ & $\mid r A / P \pm V r / T$ & $\mathrm{v}$ & * & $q V / q \pm r q / \Delta$ & $9 \Lambda / 1 \pm T r / V$ & $\mathrm{v}$ & \\
\hline Ns & $|r T /| \pm \Delta \mid / T$ & Ire $\pm \mathrm{VT} / 9$ & $\wedge$ & * & $V Y \pm r r / V$ & $V T / Y \pm T \Delta / \Delta$ & $\wedge$ & \\
\hline Ns & $\mid r \Lambda / T \pm \Delta+/ T$ & $\mid r T / r \pm r r / \Delta$ & 9 & * & $V A / T \pm r F / V$ & $v q / r \pm r v / r$ & 9 & \\
\hline Ns & $\mid r g / T \pm p q$ & $|r+| \Delta \pm \gamma \mid / q$ & 1. & Ns & $11 / r \pm r v / \Delta$ & $\Lambda T \pm r q / \Delta$ & 1. & \\
\hline \multicolumn{3}{|c|}{ Area } & & \multicolumn{3}{|c|}{$\mathrm{SD1} / \mathrm{SD2}$} & & \\
\hline Ns & $9 / 9 \pm 9$ & $9 / r \pm 9 / 9$ & 1 & Ns & $|r \Delta / \Lambda \pm r| / \Delta$ & $|r| / r \pm r \mid / \Lambda$ & 1 & \\
\hline Ns & $\mid r / q \pm 1+/ \gamma$ & $\|r / F \pm\| / \Delta$ & r & * & $19 \cdot \pm F r / \Delta$ & $19 \cdot / 9 \pm r 1 / 1$ & r & y \\
\hline Ns & $19 / r \pm 1 F / 9$ & $|\Lambda / q \pm| \gamma / 1$ & $r$ & * & $r \Delta \Lambda / P \pm \Delta Q / \varphi$ & $r \Delta V / q \pm \Delta Q / T$ & $r$ & \\
\hline Ns & $T r / q \pm 1 \wedge / T$ & $r r / v \pm r / / \Lambda$ & f & * & $r+\rho / V \pm V \wedge / \varphi$ & $r 11 / 9 \pm 9 r / 9$ & f & \\
\hline Ns & $r V / r \pm T I$ & $r V / F \pm T G / F$ & $\Delta$ & * & 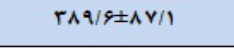 & $\varphi \cdots / v \pm \wedge r / l$ & $\Delta$ & \\
\hline Ns & $r q / q \pm r r / 1$ & $r+/ r \pm r+/ 1$ & 9 & * & $F r T / T \pm 1+p / T$ & $P Q P / G \pm q \vee / \checkmark$ & 9 & \\
\hline Ns & $r T / r \pm r F / \Lambda$ & $r r / \varphi \pm r r / s$ & $\mathrm{v}$ & * & $\Delta .9 / r \pm 119 / F$ & $\Delta r \Delta / A \pm \| 1 / / T$ & $\mathrm{v}$ & \\
\hline Ns & $r r / Y \pm r g / T$ & $r \Delta \pm r g / 1$ & $\wedge$ & * & $\Delta F G / I \pm / F T / T$ & $\Delta V Q / r \pm I r r / \Delta$ & $\wedge$ & \\
\hline Ns & $r \Delta / F \pm T V / T$ & $r q / q \pm r A / r$ & 9 & * & $910 / 1 \pm 101 / 9$ & $9 P A / V \pm 1 \Delta T / A$ & 9 & \\
\hline Ns & $r g / T \pm T A / T$ & $r v / v \pm f-$ & 1. & Ns & $G F \Delta / V \pm 1 \Lambda+/ T$ & $9 V V / V \pm 190$ & 1. & \\
\hline \multicolumn{3}{|c|}{ RQA } & ل ل ل انديس & \multicolumn{3}{|c|}{ SDXX } & & \\
\hline * & $g / v \pm r / r$ & $v / l \pm r / v$ & $\mathbf{R R}$ & Ns & $1 \cdot v /| \pm p| / 1$ & $11 \cdot \pm \Delta r / \Lambda$ & 1 & \\
\hline Ns & $\mid \gamma / q \pm p$ & $\mid \Lambda / p \pm p / p$ & قطعيت & Ns & $1-v / 1 \pm p 1 / 1$ & $\| 1 \cdot \pm \Delta r / \Lambda$ & r & \\
\hline Ns & $\| V / V \pm \mid v / v$ & ITr/9DTr/A & $\mathbf{L}$ & Ns & $1+v / 1 \pm+1 / 1$ & $11 \cdot \pm \Delta r / A$ & r & \\
\hline Ns & $r T \cdot / F \pm I F / / \Delta$ & 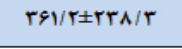 & $L \max$ & Ns & $1 \cdot v / 1 \pm F 1 / 1$ & $\| 1+ \pm \Delta T / \Lambda$ & f & \\
\hline Ns & $F p / / \pm 11$ & $P Q / T \pm I T$ & آنترويى & Ns & $1-v / 1 \pm F 1 / 1$ & $11 \cdot \pm \Delta r / \Lambda$ & $\Delta$ & \\
\hline Ns & $10 / 9 \pm 9 / \mathrm{r}$ & $19 / 1 \pm 9 / 1$ & LAM & Ns & $1 \cdot \gamma / 1 \pm p 1 / 1$ & $11= \pm \Delta r / \Lambda$ & 9 & \\
\hline Ns & $\Gamma \mid \wedge \pm 1-9 / \vee$ & $r r \Delta / 1 \pm / f q / \Lambda$ & $V \max$ & Ns & $1 \cdot v / 1 \pm p 1 / 1$ & $11 \cdot \pm \Delta r / \Lambda$ & $\mathrm{v}$ & \\
\hline Ns & $19 \cdot / V \pm F F$ & $10 r / q \pm r v / r$ & T1 & Ns & $1 \cdot v / 1 \pm F 1 / 1$ & $11+ \pm \Delta r / \Lambda$ & $\wedge$ & \\
\hline \multirow[t]{2}{*}{ Ns } & $r 9 / 9 \pm 1 / T$ & $r g / 1 \pm 1 / r$ & Trans & Ns & $1-v / 1 \pm F 1 / 1$ & $11 \cdot \pm \Delta r / A$ & 9 & \\
\hline & & & & Ns & $1-v / 1 \pm F 1 / 1$ & $11 \cdot \pm \Delta r / \Lambda$ & 1. & \\
\hline \multicolumn{3}{|c|}{ ضرايب خيندجملهاى } & إلانديس & & & & ل آنتروبىى & \\
\hline Ns & $\begin{array}{c}-1 T \pm 1 / r \Delta \times)^{-1+} \\
9 / 9 \times 1 .\end{array}$ & $\begin{array}{c}-\pi \pm 9 /-\Delta \times\left.\right|^{-"} \\
-r /|\times| .\end{array}$ & $\mathbf{A}$ & Ns & $-1 T \Delta / 9 \pm 1.9 / 9$ & $-\mid r F / 1 \pm 1+F / \Delta$ & تخمين & \\
\hline Ns & 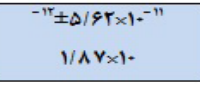 & $\begin{array}{c}{ }^{-\pi} \pm p / \cdot \gamma_{x} \mid+"= \\
q /-p \times 1 .\end{array}$ & $\mathbf{B}$ & * & $\Delta / \cdot 9 \times 1+\Delta \pm 1 / r r \times 10^{-\Delta}$ & $\Delta /-\left.|x|\right|^{-\Delta} \pm 1 / r T \times||^{-\Delta}$ & 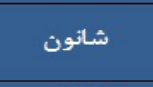 & \\
\hline Ns & $\mid \cdots \pm 1 /-9 \times 10^{-11}$ & $1 \cdots \pm \Lambda / 9 \Delta \times)^{-1 \pi}$ & $\mathbf{C}$ & Ns & 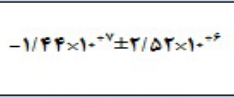 & $-1 / \% \Delta \times 1+^{-v} \pm r / \% q \times 10^{-*}$ & كَاريتمى انرثى & \\
\hline Ns & 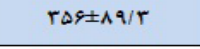 & $\mathrm{r} \Delta \mathrm{V} / \mathrm{A} \pm \mathrm{A} \mathrm{A} / \mathrm{T}$ & D & Ns & $P 9 \cdot \pm 197 / 9$ & $f F g / \Delta \pm 1 \Delta V / F$ & نمونه & \\
\hline Ns & $r r / \Lambda \pm 11 / 9$ & $r r / A \pm 1 r / 9$ & $\mathbf{E}$ & & & & & \\
\hline \multicolumn{3}{|c|}{ ضرايب DFA } & 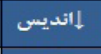 & $* *$ & $9 Q 9 / \wedge \pm \Delta \Delta / T$ & $V / 9 / 4 \pm F q / T$ & نماى ليإيانوف & \\
\hline ** & $-/ T F \times 1+^{F F} \pm T-F / \Lambda$ & $-/ T F \times \mid{ }^{-F} \pm T G P / T$ & D & Ns & $-|9| \times \mid{ }^{-F} \pm T / 1 / \Delta$ & $-|9| \times \mid{ }^{-F} \pm T r T / T$ & بيجيدكى & \\
\hline ** & $0.9 / 9 \pm T+4 / \Lambda$ & $\Delta V A / I \pm r G F / T$ & $\alpha$ & $* *$ & $T / 4 V \times 10^{-8} \pm 1 / 199 \times 10^{-8}$ & $1 / \wedge \Gamma \times\left.\right|_{0^{-8}} \pm|/ 4 \times|^{-9}$ & بعد جاسازى & \\
\hline \multicolumn{3}{|c|}{ SD2 } & ل اتأخير & \multicolumn{3}{|c|}{ SD1 } & 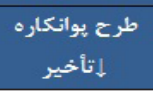 & \\
\hline
\end{tabular}




\begin{tabular}{|c|c|c|c|c|c|c|c|}
\hline Ns & $\mid F \wedge / r \pm \Delta V / \Delta$ & $\mid f q / F \pm 9 \Delta / 1$ & 1 & * & $19 \pm 0$ & $1 N / r \pm r / V$ & 1 \\
\hline Ns & $\mid F V / \Delta \pm \Delta \varphi / 9$ & $\mid F \wedge / V \pm \varphi \Delta / \Delta$ & $r$ & * & $r F \pm 1 T / T$ & $r r / l \pm V / r$ & r \\
\hline Ns & $\mid F F / 9 \pm \Delta \Delta / Q$ & $\mid f 9 \pm 90 / 4$ & $r$ & * & $r v / r \pm 19 / r$ & $r g / 1 \pm 1+/ T$ & $r$ \\
\hline Ns & $\mid F T / \Delta \pm \Delta F / 9$ & $\mid F r / q \pm 9 F / \Lambda$ & $F$ & * & $F F / 1 \pm r / / 1$ & $p r / p \pm 1 \% / 1$ & $f$ \\
\hline Ns & $1 r \wedge / q \pm \Delta r / V$ & $\mid f+/ 1 \pm 9+/ 0$ & $\Delta$ & * & $\Delta P / r \pm r r / Q$ & $\Delta r / q \pm 19 / 9$ & $\Delta$ \\
\hline Ns & $\mid r g / \Delta \pm \Delta r / F$ & $\mid r v / P \pm 9 r / A$ & 9 & * & $\Delta Q / \Lambda \pm r \xi / 9$ & $9 \cdot / r \pm r+1 r$ & 9 \\
\hline Ns & $|r r / V \pm \Delta| / \varphi$ & $\mid r r / r \pm g r / 0$ & $\mathrm{~V}$ & * & GV/G士rq & $9 N / \Delta \pm T r / 9$ & V \\
\hline Ns & $\mid r+/ p \pm \Delta+/ T$ & $\mid r+/ \Lambda \pm 9 T / V$ & $\wedge$ & * & $r \mid / q \pm r r / r$ & $V T / Q \pm T \Delta / r$ & $\wedge$ \\
\hline Ns & $\mid r g / \Delta \pm p q / r$ & $\mid r V \pm g r / F$ & 9 & * & $v \wedge \pm r r / 1$ & $v q \pm r v / 1$ & 9 \\
\hline Ns & $\mid T F / \Delta \pm F V / \Lambda$ & $\mid T \Delta / T \pm 91 / 9$ & 1. & * & $\Lambda+/ q \pm r q / 9$ & $\Lambda 1 / Y \pm r q / T$ & 1. \\
\hline \multicolumn{3}{|c|}{ Area } & & \multicolumn{3}{|c|}{$\mathrm{SD} 1 / \mathrm{SD} 2$} & \\
\hline Ns & $9 / \Delta \pm \Delta / 9$ & $q \pm 9$ & 1 & Ns & $|r v / r \pm r| / 9$ & $\mid r F / 1 \pm r r / r$ & 1 \\
\hline Ns & $|r / \Lambda \pm|+/ Y$ & $\mid r \pm 1+19$ & $r$ & * & $|91 / p \pm F| / T$ & $19 r / 9 \pm r \Lambda / r$ & $r$ \\
\hline Ns & $19 / 1 \pm 1 \% / 1$ & $1 \Lambda / T \pm 1 Q / V$ & $r$ & Ns & $r \varphi+/ \Delta \pm \Delta 9 / T$ & $r G T / I \pm F V / V$ & $r$ \\
\hline Ns & $r r / 9 \pm 11 / r$ & $r \mid / \Lambda \pm r+/ 1$ & $F$ & * & $r \cdot V \pm V r / q$ & $r \mid g / \Delta \pm \Delta V / 1$ & $f$ \\
\hline Ns & $r q / 9 \pm r \mid$ & $r g / r \pm r r / 1$ & $\Delta$ & * & $\mathrm{rar/q \pm VQ/9}$ & $P \cdot V / r \pm V \varphi / T$ & $\Delta$ \\
\hline Ns & $r q / T \pm r r / T$ & $r q / 1 \pm r v / \Delta$ & 9 & * & $p r g / r \pm q F / q$ & $F 9 / / \wedge \pm \wedge \wedge / \vee$ & 9 \\
\hline Ns & $r / / q \pm r p / q$ & $r T \pm r+10$ & $\mathrm{~V}$ & $* *$ & $\Delta \mid r / V \pm 1+\Lambda / P$ & $\Delta F \Delta / T \pm 1-\Lambda / V$ & $\mathrm{v}$ \\
\hline Ns & $r r / r \pm r g / r$ & rr/g士rr/A & $\wedge$ & ** & $\Delta F Q / T \pm I T Q / T$ & $\Delta \wedge q / F \pm|r| / r$ & $\wedge$ \\
\hline Ns & $r \Delta \pm r v / \theta$ & $r \Delta / \varphi \pm r+/ \gamma$ & 9 & * & $919 / T \pm / F T / 9$ & $991 / \mathrm{T} \pm 16-/ 4$ & 9 \\
\hline Ns & $r \Delta / v \pm r \Lambda / \varphi$ & $r g / 1 \pm r g / 1$ & 1. & * & SPN/DEIGT/F & $991 / r \pm 10-/ 1$ & 1. \\
\hline \multicolumn{3}{|c|}{ RQA } & 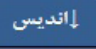 & \multicolumn{3}{|c|}{ SDXX } & \\
\hline Ns & $9 / 9 \pm T / T$ & $v \pm r / Q$ & RR & Ns & $1-\Delta / \Lambda \pm \psi / \gamma$ & $1.9 / 0 \pm F 9 / 0$ & 1 \\
\hline Ns & $|v / v \pm p /|$ & $1 \Lambda / 1 \pm F / T$ & قطعيت & Ns & $1-\Delta / \Lambda \pm \psi / V$ & $1.9 / 0 \pm F 9 / 0$ & r \\
\hline Ns & $11 \gamma / r \pm 1 v / 9$ & $\mid r I / \Lambda \pm r \Lambda / F$ & $\mathbf{L}$ & Ns & $1-\Delta / \Lambda \pm \psi / / \gamma$ & $1.910 \pm p 9 / 0$ & $r$ \\
\hline Ns & $r|g / v \pm| f \mid$ & $r F v / r \pm r / g / 9$ & $L \max$ & Ns & $1-\Delta / \Lambda \pm \varphi+/ \gamma$ & $1.9 / 0 \pm F 9 / 0$ & f \\
\hline Ns & $F r / v \pm 11 / r$ & $F \Delta / \Delta \pm 1 T / 9$ & 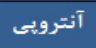 & Ns & $1+\Delta / \Lambda \pm \psi+/ \gamma$ & $1.910 \pm p 910$ & $\Delta$ \\
\hline Ns & $10 / r \pm 9 / \%$ & $10 / Y \pm 9 / 1$ & LAM & Ns & $1-\Delta / A \pm \psi+/ \gamma$ & $1.9 / 0 \pm F 9 / 0$ & 9 \\
\hline Ns & $r \mid F / r \pm \| 1 /=/ T$ & rTVEIrq/T & $V \max$ & Ns & $1+\Delta / \Lambda \pm \varphi+/ \gamma$ & $1.9 / 0 \pm F 9 / 0$ & $\mathrm{v}$ \\
\hline Ns & $19 r \pm p 910$ & $109 / 4 \pm r q$ & T1 & Ns & $1-\Delta / \Lambda \pm p+/ \gamma$ & $1.9 / 0 \pm p 9 / 0$ & $\wedge$ \\
\hline \multirow[t]{2}{*}{ Ns } & $r 9 / 9 \pm 1 / r$ & $r g / A \pm 1 / r$ & Trans & Ns & $1+\Delta / A \pm \varphi+/\rangle$ & $1.910 \pm F 9 / 0$ & 9 \\
\hline & & & & Ns & $1-\Delta / \Lambda \pm \psi-/ \gamma$ & $1.9 / 0 \pm p 9 / 0$ & 1. \\
\hline \multicolumn{3}{|c|}{ ضرايب بِندجملهاى } & 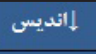 & & & & ل آنترويهى \\
\hline Ns & $\begin{array}{c}\left.{ }^{-1} \pm 1 / 49 \times\right)^{-1+} \\
1 / \Delta 9 x)^{-}\end{array}$ & $\begin{array}{c}-1 \pi \pm 1 / \cdot r \times)^{-1+} \\
\Delta / 9 \Lambda \times)^{-}\end{array}$ & $\mathbf{A}$ & Ns & $-|r r \pm| \cdot r / r$ & $-\mid r F / 9 \pm 1+9 / 1$ & تخمين \\
\hline * & $\begin{array}{c}-1 r \pm 9(\Delta \Delta \times){ }^{-11} \\
9|-\Delta \times| .\end{array}$ & 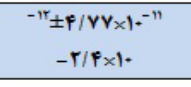 & B & Ns & $\Delta / \cdot q \times 10^{-\Delta} \pm 1 / T P \times 1+\Delta$ & $\Delta /-9 \times 10^{-\Delta} \pm 1 / \mid 9 \times 10^{-\Delta}$ & 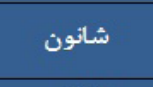 \\
\hline Ns & $1 \cdots \pm 1 /|f \times|^{-n}$ & $\mid \cdots \pm q / 99 \times 1)^{-1 r}$ & C & Ns & $-1 / 4 p \times 10^{-4} \pm r / r q \times 10^{-4}$ & $-1 / p r \times 1+^{-v} \pm r / \Delta \times 1+^{-4}$ & لثاريتمى اثرثى \\
\hline Ns & $r \Delta \xi / q \pm \wedge \vee / \Lambda$ & $\mathrm{rSl} / \mathrm{A} \pm \mathrm{rV} / \mathrm{q}$ & D & Ns & $\mathrm{FAT} / \mathrm{V} \pm 1 \Delta 9 / \Delta$ & PQP/GIIGT/A & نمونه \\
\hline Ns & $r r / A \pm 11 / \Delta$ & $r F / r \pm 11$ & $\mathbf{E}$ & & & & \\
\hline \multicolumn{3}{|c|}{ ضر ايب DFA } & 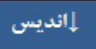 & $\dot{*}$ & $99 \Lambda / 9 \pm \Delta F / 9$ & $V / V / \Lambda \pm F Q / T$ & نماى ليإِانوف \\
\hline * & $\cdot / T f \times\left|{ }^{-F} \pm\right| \gamma \gamma / \Lambda$ & $-/ T F \times 1 \cdot{ }^{-F} \pm T r \Lambda / P$ & D & Ns & $\cdot|9| \times \mid{ }^{-F} \pm r \cdot \Delta / r$ & $-|9| \times 1 \cdot^{-F} \pm r \mid f / 1$ & "يجيد \\
\hline * & $\Delta * / T \pm \mid \gamma \vee / \Lambda$ & $\Delta F T / Q \pm T r A / F$ & $\alpha$ & $* *$ & 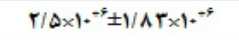 & 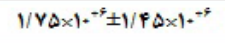 & بعد جاسازى \\
\hline
\end{tabular}


1. Walker MP, Harvey AG. Obligate symbiosis: sleep and affect. Sleep Med Rev. 2010; 14(4): 215-7.

2. Baloochi Anaraki M, Rahimi S. Dream and sleep disorders: psychological and pathophysiological aspects. Shefaye Khatam. 2015; 3: 99-111.

3. Astill RG, Van der Heijden KB, Van Ijzendoorn $\mathrm{MH}$, Van Someren EJ. Sleep, cognition, and behavioral problems in school-age children: a century of research meta-analyzed. Psychol Bull. 2012; 138(6): 1109-38.

4. Lim J, Dinges DF. A meta-analysis of the impact of short-term sleep deprivation on cognitive variables. Psychol Bull. 2010; 136(3): 375-89.

5. Eshaghabadi A, Sahab Negah S. Sleepiness in road accidents. Shefaye Khatam. 2016; 3(4): P13.

6. Palmer CA, Alfano CA. Sleep and emotion regulation: an organizing, integrative review. Sleep Med Rev. 2016; 31: 6-16.

7. Dinges DF, Pack F, Williams K, Gillen KA, Powell JW, Ott GE, et al. Cumulative sleepiness, mood disturbance, and psychomotor vigilance performance decrements during a week of sleep restricted to 4-5 hours per night. Sleep. 1997; 20(4): 267-77.

8. Alfano CA, Ginsburg GS, Kingery JN. Sleep-related problems among children and adolescents with anxiety disorders. J Am Acad Child Adolesc Psychiatry. 2007; 46(2): 224-32.

9. Franzen PL, Siegle GJ, Buysse DJ. Relationships between affect, vigilance, and sleepiness following sleep deprivation. J Sleep Res. 2008; 17(1): 34-41.

10. Talbot L, McGlinchey E, Kaplan K, Dahl R, Harvey A. Sleep deprivation in adolescents and adults: changes in affect. Emotion. 2010; 10(6): 831-41.

11. Berger R, Miller A, Seifer R, Cares S, Lebourgeois M. Acute sleep restriction effects on emotion responses in 30- to 36-month-old children. J Sleep Res. 2012; 21(3): 235-46.

12. Minkel J, Htaik O, Banks S, Dinges D. Emotional expressiveness in sleep deprived healthy adults. Behav Sleep Med. 2011; 9(1): 5-14.

13. Franzen P, Buysse D, Dahl R, Thompson W, Siegle G. Sleep deprivation alters pupillary reactivity to emotional stimuli in healthy young adults. Biol Psychol. 2009; 80(3): 300-5.

14. Dahl R, Lewin D. Pathways to adolescent health sleep regulation and behavior. J Adolesc Health. 2002; 31(6): 175-84.
15. Yoo S, Hu P, Gujar N, Jolesz F, Walker M. A deficit in the ability to form new human memories without sleep. Nat Neurosci. 2007; 10(3): 385-92.

16. Gujar N, Yoo S, Hu P, Walker M. Sleep deprivation amplifies reactivity of brain reward networks, biasing the appraisal of positive emotional experiences. J Neurosci. 2011; 31(12): 4466-74.

17. Swann C, Yelland G, Redman J, Rajaratnam S. Chronic partial sleep loss increases the facilitatory role of a masked prime in a word recognition task. J Sleep Res. 2006; 15(1): 23-9.

18. Pessoa L, Adolphs R. Emotion processing and the amygdala: from a 'low road' to 'many roads' of evaluating biological significance. Nature Rev Neurosci. 2010; 11(11): 773-83.

19. Whalen P, Rauch S, Etcoff N, McInerney S, Lee M, Jenike M. Masked presentations of emotional facial expressions modulate amygdala activity without explicit knowledge. J Neurosci. 1998; 18: 411-8.

20. Ogawa Y, Kanbayashi T, Saito Y, Takahashi Y, Kitajima T, Takahashi K, et al. Total sleep deprivation elevates blood pressure through arterial baroreflex resetting: a study with microneurographic technique. Sleep Med. 2003; 26(8): 986-9.

21. Goshvarpour A, Abbasi A, Goshvarpour A. Evaluating autonomic parameters: the role of sleep duration in emotional responses to music. Iran J Psychiatry. 2016; 11(1): 59-63.

22. Demaree H, Schmeichel B, Robinson J, Everhart D. Behavioural, affective, and physiological effects of negative and positive emotional exaggeration. Cogn Emot. 2004; 18: 1079-97.

23. Picard RW, Vyzas E, Healey J. Toward machine emotional intelligence: analysis of affective physiological state. IEEE Trans Pattern Anal Mach Intell. 2001; 23(10): $1175-91$.

24. Kim K, Bang S, Kim S. Emotion recognition system using short-term monitoring of physiological signals. Med Biol Eng Comput. 2004; 42(3): 419-27.

25. Kreibig S, Wilhelm F, Roth W, Gross J. Cardiovascular, electrodermal, and respiratory response patterns to fear and sadness-inducing films. Psychophysiology. 2007; 44(5): 787-806.

26. Blechert J, Lajtman M, Michael T, Margraf J, Wilhelm F. Identifying anxiety states using broad sampling and advanced processing of peripheral physiological information. Biomed Sci Instrum. 2006; 42: 136-41. 


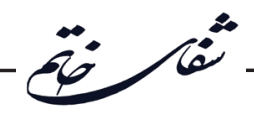

27. Yoon H, Chung S. EEG-based emotion estimation using Bayesian weighted-log-posterior function and perceptron convergence algorithm. Comput Biol Med. 2013; 43(12): 2230-7.

28. Li C, Feng Z, Xu C. Error-correcting output codes for multi-label emotion classification. Multimed Tools Appl. 2016; 75(22): 14399-416.

29. Aftanas LI, Lotova NV, Koshkarov VI, Makhnev VP, Mordvintsev YN, Popov SA. Non-linear dynamic complexity of the human EEG during evoked emotions. Int J Psychophysiol. 1998; 28(1): 63-76.

30. Aftanas LI, Lotova NV, Koshkarov VI, Pokrovskaja VL, Popov SA, Makhnev VP. Non-linear analysis of emotion EEG: calculation of Kolmogorov entropy and the principal Lyapunov exponent. Neurosci Lett. 1997; 226(1): 13-6.

31. Zheng WL, Lu BL. Investigating critical frequency bands and channels for EEG-based emotion recognition with deep neural networks. IEEE Trans Auton Ment Dev. 2015; 7(3): 162-75.

32. Wang L, Liu G, Yang Z. The emotion recognition for grief based on nonlinear features of GSR. J Comput Inform Syst. 2014; 10: 1639-49.

33. Roque A, Valenti V, Guida H, Campos M, Knap A, Vanderlei L, et al. The effects of auditory stimulation with music on heart rate variability in healthy women. Clinics. 2013; 68: 960-7.

34. Silva S, Guida H, SantosAntonio A, Vanderlei L, Ferreira L, de Abreu L, et al. Auditory stimulation with music influences the geometric indices of heart rate variability in men. Int Arch Med. 2014; 7: 1-7.

35. Nardelli M, Valenza G, Greco A, Lanata A, Scilingo EP. Recognizing emotions induced by affective sounds through heart rate variability. IEEE Trans Affect Comput. 2015; 6(4): 385 - 94.

36. Lang PJ, Bradley MM, Cuthbert BN. International affective picture system (IAPS): technical manual and affective ratings. International Affective Picture System (IAPS). 1997.

37. Goshvarpour A, Abbasi A, Goshvarpour A. Affective visual stimuli: characterization of the picture sequences impacts by means of nonlinear approaches. Basic Clin Neurosci. 2015; 6(4): 209-21.

38. Proakis JG, Manolakis DG. Digital signal processing principles, algorithms, and applications. 3rd ed. New Jersey: Prentice-Hall. 1996.

39. Lempel A, Ziv J. On the complexity of finite sequences. IEEE Trans Inf Theory. 1976; 22(1): 75-81.

40. Zhang XS, Zhu YS. Detecting ventricular tachycardia and fibrillation by complexity measure. IEEE Trans Biomed Eng. 1999; 46(5): 548-55.

41. Peng CK, Buldyrev SV, Havlin S, Simons M, Stanley HE, Goldberger AL. Mosaic organization of DNA nucleotides. Phys Rev E. 1994; 49(2): 1685-9.

42. Takens F. Detecting strange attractor in turbulence. Berlin: Springer. 1980. p. 366-81.

43. Pincus SM. Approximate entropy as a measure of system complexity. Proc Natl Acad Sci USA. 1991; 88(6): 2297-301.

44. Tulppo MP, Makikallio TH, Takala TE, Seppanen T, Huikuri HV. Quantitative beat to beat analysis of heart rate dynamics during exercise. Am J Physiol. 1996; 271(1): H244-H52.

45. Guzik P, Piskorski J, Krauze T, Schneider R, Wesseling K, Wykretowicz A, et al. Correlations between the poincare plot and conventional heart rate variability parameters assessed during paced breathing. J Physiol Sci. 2007; 57(1): 63-71.

46. Piskorski J, Guzik P. Filtering poincare plots. Computational Methods in Science and Technology. 2005; 11(1): 39-48.

47. Rosenstein MT, Collins JJ, DeLuca CJ. A practical method for calculating largest lyapunov exponents from small data sets. Physica D: Nonlinear Phenomena. 1993; 65(1-2): 117-34.

48. Marwan N, Romano M, Thiel M, kurths J. Recurrence plots for the analysis of complex systems. Phys Rep. 2007; 438(5-6): 237-329.

49. Moharreri S, Rezaei S, Dabanloo N, Parvaneh S. Extended parabolic phase space mapping (EPPSM): novel quadratic function for representation of heart rate variability signal. Comput Cardiol. 2014; 41: 417-20.

50. Kreibig SD. Autonomic nervous system activity in emotion: a review. Biol Psychol. 2010; 84(3): 394-421.

51. Jerritta S, Murugappan M, Wan K, Yaacob S. Classification of emotional states from electrocardiogram signals: a non-linear approach based on hurst. Bio Med Eng Online. 2013; 12(44): Doi: 10.1186/1475-925X-1244.

52. Valenza G, Allegrini P, Lanata A, Scilingo EP. Dominant lyapunov exponent and approximate entropy in heart rate variability during emotional visual elicitation. Front Neuroeng. 2012; 5: 1-7.

53. Valenza G, Citi L, Lanata A, Scilingi E, Barbieri R. Revealing real-time emotional responses: a personalized assessment based on heartbeat dynamics. Sci Rep. 2014; 4(4998). Doi:10.1038/srep04998. 